\title{
Remodeling a DS-diagram into one with E-cycle
}

\author{
Hiroshi IKEDA, Masakatsu YAMASHITA and Kazuo YOKOYAMA
}

Kobe University, Toyo University and Sophia University

\section{Introduction}

B. G. Casler constructed a standard spine for a 3-manifold with boundary from the polyhedral structure, in [1]. He stated there that two 3-manifolds are homeomorphic if and only if they have a standard spine in common. Standard spines form a good subclass of the spines of 3-manifolds. Later, in [7], Ishii found a better class of spines for closed 3-manifolds. He constructed a spine by making use of a flow on the manifold and called such a spine a flow-spine. Spines of a closed manifold are understood to be the usual ones of the manifold from which a small ball is removed. It is known that the flow-spine form a good subclass of the standard spines. In this paper, we exhibit an algorithm to deform a standard spine to a flow-spine in the given closed manifold by a combinatorial topological method. It is, however, hard to see directly whether a standard spine is a flow-spine or not. By DS-diagrams (see Definition 1.1), we get rid of the difficulty. It is known in [5] that any closed 3-manifold has a DS-diagram constructed from a standard spine. The flow-spines correspond to the DS-diagrams with Ecycle, see [4] and [8]. Thus the problem above can be translated into the remodeling problem of a DS-diagram into one with E-cycle (see Definition 2.2).

The main theorem of this paper can be stated as follows (see Definition 1.2 for the notion of DS-isomorphism).

THEOREM 1.1. Any DS-diagram is DS-isomorphic to a DS-diagram with E-cycle.

We prove this theorem by finding a DS-isomorphism to get a DS-diagram with E-cycle algorithmically.

Including the concept of DS-isomorphism, let us review briefly some of the definitions made in [4] through [8] to understand the theorem.

Consider a 2-sphere $S^{2}$ and a connected 3-regular graph $G$ embedded in $S^{2}$. Let $V_{G}$ be the set of vertices of $G$. Then $G$ induces a natural structure of cell complex $K(G)$ on $S^{2}$; 0-cells are elements of $V_{G}, 1$-cells are the connected components of $G-V_{G}$ and 2-cells are the connected components of $S^{2}-G$. For a definition of cell complexes, see for example, [9].

DEFINITION 1.1. A triple $\Delta=\left(S^{2}, G, f\right)$ is called a $D S$-diagram if 
(1) $G$ is a connected 3-regular graph embedded in $S^{2}$.

(2) For a polyhedron $P$ with cell structure $K(P), f$ is a continuous map from $S^{2}$ onto $P$. $f$ is called an identification map of $\Delta$,

(3) $f: K(G) \rightarrow K(P)$ is a cellular map, that is, for each $\sigma \in K(G),\left.f\right|_{\sigma}$ is a homeomorphism from $\sigma$ onto a cell $\lambda=f(\sigma)$ of $K(P)$,

(4) for each $k$-cell $\lambda^{k} \in K(P), \sharp f^{-1}\left(\lambda^{2}\right)=2, \sharp f^{-1}\left(\lambda^{1}\right)=3$ and $\sharp f^{-1}\left(\lambda^{0}\right)=4$, where $\sharp f^{-1}\left(\lambda^{k}\right)$ means the number of the connected components of $f^{-1}\left(\lambda^{k}\right)$.

We understand that the cells of $K(G)$ and $K(P)$ are oriented so that $f$ is orientation preserving. For each cell $\sigma \in K(G)$, we call the oriented cell $f(\sigma) \in K(P)$ a label of $\sigma$. We often say that $f(\sigma)$ is a $k$-label of $\sigma$ if $\operatorname{dim} \sigma=k$. Usually we say $\sigma \in K(G)$ a cell in $\Delta$ and $f(\sigma) \in K(P)$ a label in $\Delta$.

Let $\Delta=\left(S^{2}, G, f\right)$ be a DS-diagram with an identification map $f: S^{2} \rightarrow P$. The identification space $S^{2} / f=P$ is necessarily a closed fake surface (for the definition of a closed fake surface, see [2]). Let $B^{3}$ be a 3-ball with boundary $\partial B^{3}=S^{2}$. Then the identification space $B^{3} / f$ is automatically a closed 3-manifold. We will denote $B^{3} / f$ by $M(\Delta)$ and call it the manifold associated with the DS-diagram $\Delta$.

We explain here the terminology "DS-isomorphism" briefly, see [6] for detail. It should be remarked that if $\Delta^{\prime}$ is DS-isomorphic to $\Delta$, then a manifold $M\left(\Delta^{\prime}\right)$ associated with $\Delta^{\prime}$ is homeomorphic to $M(\Delta)$. It is not hard to see that the replacements stated below correspond to well-known deformations of a spine keeping the manifold fixed.

DEFINITION 1.2. Let $v$ be a 0-label of a DS-diagram $\Delta_{1}$ and

$$
\Sigma_{1}(v)=\left\{A^{+} B^{+}, C^{+} D^{+}, A^{+} C^{+}, B^{+} D^{+}, A^{+} D^{+}, B^{+} C^{+}\right\}
$$

the surroundings around $v$ in $\Delta_{1} ; A^{+}$means the head part of an arrow indicating a 1-label $A$. We can consider three pairs $\left\{A^{+} B^{+}, C^{+} D^{+}\right\},\left\{A^{+} C^{+}, B^{+} D^{+}\right\},\left\{A^{+} D^{+}, B^{+} C^{+}\right\}$. Choose one of them, say $\left\{A^{+} B^{+}, C^{+} D^{+}\right\}$. Replacing $\Sigma_{1}(v)$ by

$$
\begin{aligned}
\Sigma_{2}\left(E F^{-1}, G H^{-1}\right)=\{ & E F^{-1}, G H^{-1} ; A^{+} B^{+}, C^{+} D^{+} \\
& \left.A^{+} G E C^{+}, B^{+} G F C^{+}, A^{+} H E D^{+}, B^{+} H F D^{+}\right\},
\end{aligned}
$$

we obtain another DS-diagram $\Delta_{2}$ from $\Delta_{1}$. Then $\Delta_{1} \Leftrightarrow \Delta_{2}$ is called an elementary deformation of Type I (or briefly, I-deformation, see Figure 1-a). We use the notation $\Phi=$ $\Phi\left(A^{+} B^{+}, C^{+} D^{+}\right): \Delta_{1} \Rightarrow \Delta_{2}$ and $\Phi^{-1}=\Phi^{-1}\left(E F^{-1}, G H^{-1}\right): \Delta_{2} \Rightarrow \Delta_{1}$. We say $\Phi$ is of type $I^{+}$and $\Phi^{-1}$ is of type $I^{-}$.

Let $A$ be a 1-label of a DS-diagram $\Delta_{3}$ with surroundings

$$
\Sigma_{3}(A)=\left\{P^{+} A S^{-}, Q^{+} A T^{-}, R^{+} A U^{-}, Q^{+} R^{+}, R^{+} P^{+}, P^{+} Q^{+}, T^{-} U^{-}, U^{-} S^{-}, S^{-} T^{-}\right\} ;
$$

$P^{+}$(or $S^{-}$) means the head part (or the tail part) of an arrow indicating a 1-label $P$ (or $S$, respectively), and so on. 

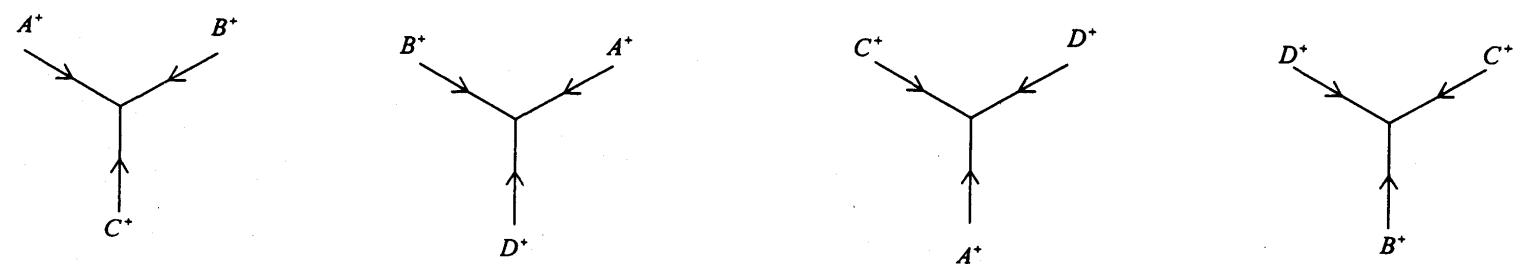

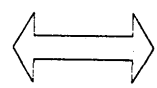
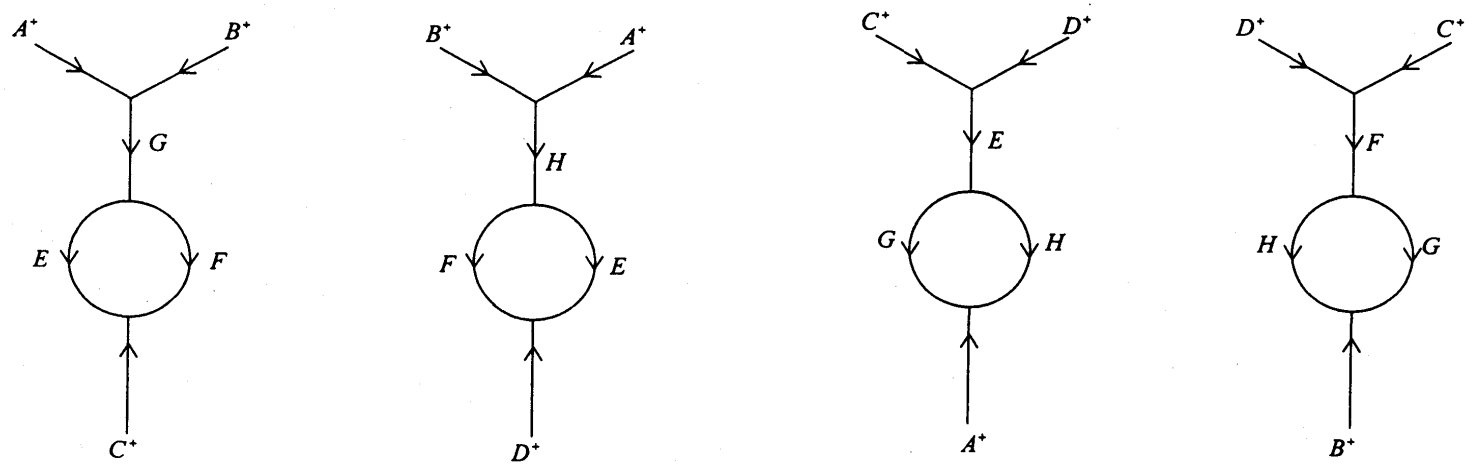

FIGURE 1-a. Elementary deformation of type I.
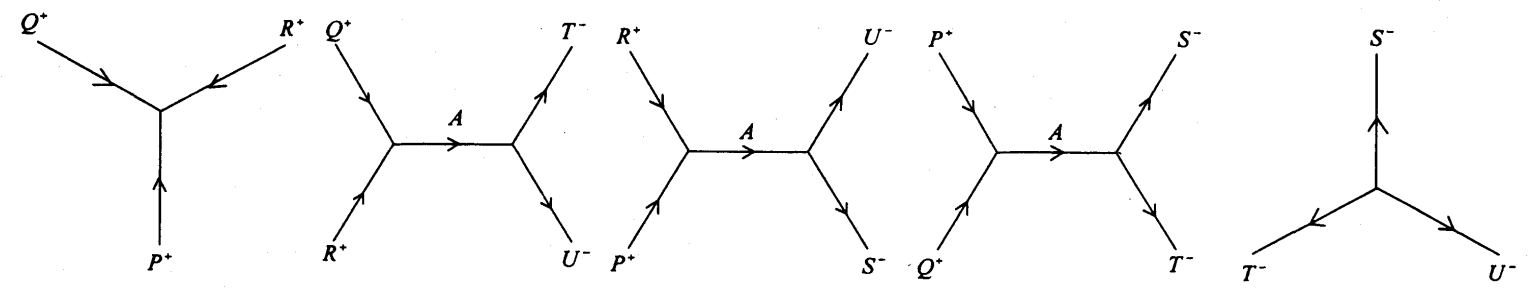

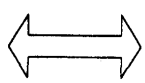
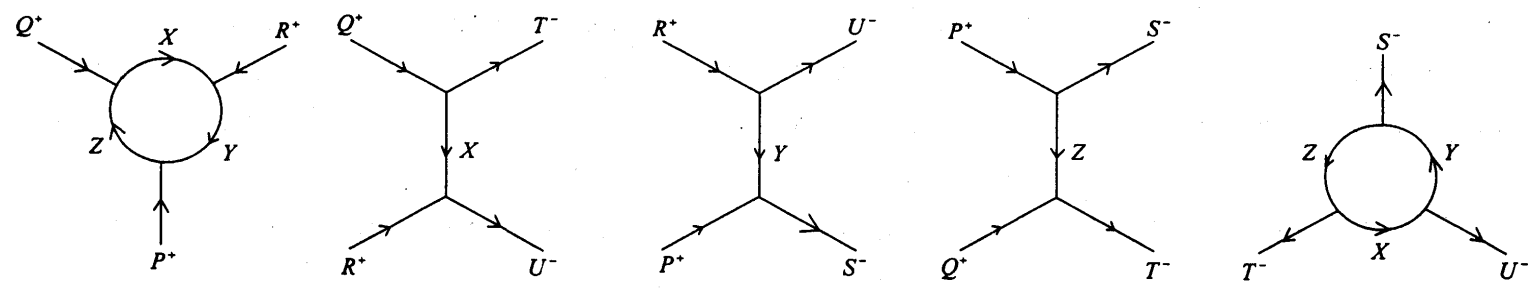

FIGURE 1-b. Elementary deformation of type II. 

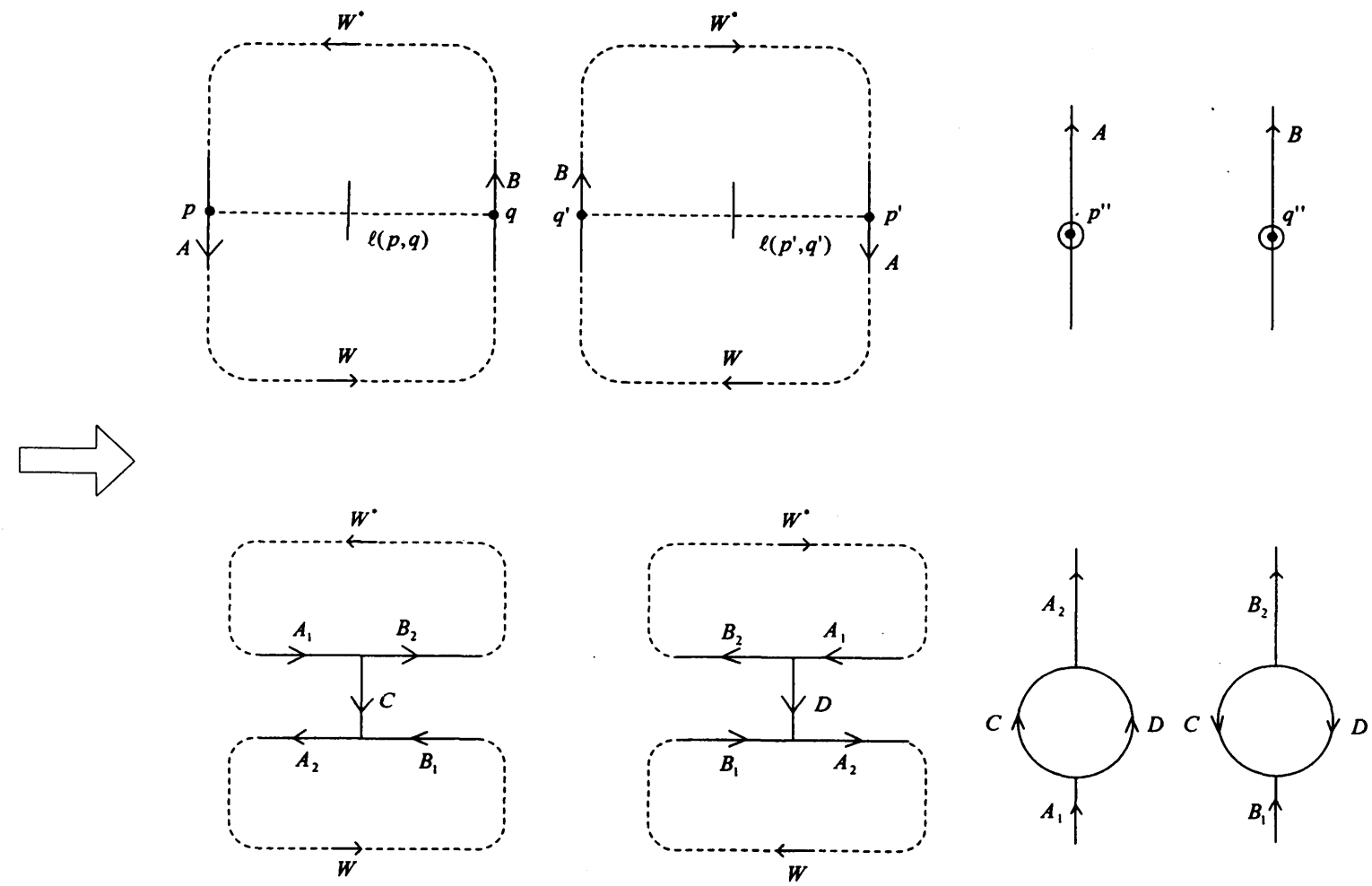

FIGURE 2. Piping.

Suppose $\Delta_{4}$ be obtained from $\Delta_{3}$ by only replacing only $\Sigma_{3}(A)$ by

$$
\begin{aligned}
\Sigma_{4}(X Y Z)= & \left\{X Y Z ; P^{+} S^{-}, Q^{+} T^{-}, R^{+} U^{-}, Q^{+} X R^{+}, R^{+} Y P^{+},\right. \\
& \left.P^{+} Z Q^{+}, T^{-} X U^{-}, U^{-} Y S^{-}, S^{-} Z T^{-}\right\} .
\end{aligned}
$$

Then $\Delta_{3} \Leftrightarrow \Delta_{4}$ is called an elementary deformation of type II (or briefly, II-deformation, see Figure 1-b). We use the notation $\Psi=\Psi(A): \Delta_{3} \Rightarrow \Delta_{4}$ and $\Psi^{-1}=\Psi^{-1}(X Y Z): \Delta_{4} \Rightarrow$ $\Delta_{3}$. We say $\Psi$ is of type $I I^{+}$and $\Psi^{-1}$ is of type $I I^{-}$.

A 1-label said to be of loop-type if the closure is a loop, and of arc-type otherwise. We note that II-deformation is available if $A, X, Y, Z$ are all 1-labels of arc-type.

A finite application of elementary deformations is called a $D S$-deformation. Suppose $\Delta$ and $\Delta^{\prime}$ are DS-diagrams. We say $\Delta^{\prime}$ is DS-isomorphic to $\Delta$ if $\Delta^{\prime}$ is obtained from $\Delta$ by a DS-deformation.

The DS-isomorphism, called a piping, on a DS-diagram plays an important role in this paper. We explain here this deformation.

DEFINITION 1.3. Let $\Delta=\left(S^{2}, G, f\right)$ be a DS-diagram. Let $\alpha^{+}$and $\alpha^{-}$be 2-cells in $\Delta$ with the same 2-label $\alpha$. Choosing 1-cells $P, Q$ (possibly $P=Q$ ) on the boundary $\partial \alpha^{+}$ of $\alpha^{+}$, we can denote $\partial \alpha^{+}$as

$$
\partial \alpha^{+}=P \tau_{1} \tau_{2} \cdots \tau_{n} Q \tau_{1}^{*} \tau_{2}^{*} \cdots \tau_{m}^{*}
$$


where $\tau_{i}, \tau_{j}^{*}$ are 1-cells on $\partial \alpha^{+}$. Choose two points $p \in P$ and $q \in Q$ so that $f(p) \neq f(q)$. Let $\ell^{+}(p, q)$ be a proper arc in a 2-cell $\alpha^{+}$joining $p$ with $q$. Put $x=f(p), y=f(q)$ and $\ell(x, y)=f\left(\ell^{+}(p, q)\right)$. Let $A, B, J_{i}, J_{j}^{*}$ be 1-labels of $P, Q, \tau_{i}, \tau_{j}^{*}$ respectively; that is, $A=f(P), B=f(Q), J_{i}=f\left(\tau_{i}\right), J_{j}^{*}=f\left(\tau_{j}^{*}\right)$. Then we can write

$$
\partial \alpha=A J_{1} J_{2} \cdots J_{n} B J_{1}^{*} J_{2}^{*} \cdots J_{m}^{*}=A w B w^{*},
$$

where $w=J_{1} J_{2} \cdots J_{n}$ and $w^{*}=J_{1}^{*} J_{2}^{*} \cdots J_{m}^{*}$. Then, the surroundings around $A \cup B$ are

$$
\Sigma(A \cup B)=\left\{A w B w^{*} ; \cdots A \cdots, \cdots B \cdots, \cdots A \cdots, \cdots B \cdots\right\} .
$$

Consider a DS-diagram $\Delta^{\prime}$ obtained from $\Delta$ by replacing $\Sigma(A \cup B)$ by

$$
\begin{aligned}
\Sigma^{\prime}= & \left\{C D^{-1}, A_{2} w B_{1}, B_{2} w^{*} A_{1} ; A_{1} C A_{2} \cdots, \cdots B_{1} C^{-1} B_{2} \cdots,\right. \\
& \left.\cdots A_{1} D A_{2} \cdots, \cdots B_{1} D^{-1} B_{2} \cdots\right\}
\end{aligned}
$$

We call $L=L(A, B): \Delta \Rightarrow \Delta^{\prime}$ a piping along $\ell(x, y)$, see Figure 2. We showed in [6] the fact that a manifold $M\left(\Delta^{\prime}\right)$ associated with $\Delta^{\prime}$ is homemorphic to $M(\Delta)$. Suppose that there exists a 2-gon on a DS-diagram just like $C D^{-1}$ in $\Sigma^{\prime}$. Then we can consider the inverse $L^{-1}: \Delta^{\prime} \Rightarrow \Delta$. We often use the notation $\delta\left(C D^{-1}\right)$ instead of $L^{-1}$, and call it a 2-gon collapsing. For detail, see [6].

\section{Remodeling a DS-diagram into a splittable one.}

The main purpose of this section is to show the following.

THEOREM 2.1. For any DS-diagram $\Delta$, there exists a splittable DS-diagram (see Definition 2.1 for "splittable") which is DS-isomorphic to $\Delta$.

Let $\Delta=\left(S^{2}, G, f\right)$ be a DS-diagram. Consider a pair of 2-cells in $\Delta$ with the same label $\alpha$. We denote one of them $\alpha^{+}$and the other $\alpha^{-}$. In this way, we can separate whole 2-cells in $\Delta$ into two classes $\left\{\alpha_{1}^{+}, \alpha_{2}^{+}, \cdots, \alpha_{n+1}^{+}\right\}$and $\left\{\alpha_{1}^{-}, \alpha_{2}^{-}, \cdots, \alpha_{n+1}^{-}\right\}$.

DEFINITION 2.1. The closure $Z^{+}$of $\alpha_{1}^{+} \cup \alpha_{2}^{+} \cup \cdots \cup \alpha_{n+1}^{+}$(or $Z^{-}$of $\alpha_{1}^{-} \cup \alpha_{2}^{-} \cup \cdots \cup$ $\alpha_{n+1}^{-}$) is called the positive zone (or the negative zone, respectively). We will call $\left(Z^{+}, Z^{-}\right)$a bicoloring of the DS-diagram $\Delta$. We will call $\left(Z^{+}, Z^{-}\right)$a split bicoloring of $\Delta$ if both of $Z^{+}$ and $Z^{-}$are connected. A DS-diagram $\Delta$ is splittable if $\Delta$ has a split bicoloring.

DEFINITION 2.2. Let $\Delta$ be a splittable DS-diagram with a split bicoloring $\left(Z^{+}, Z^{-}\right)$. Let $a_{1}, a_{2}, \cdots, a_{m}$ be a sequence of 1-cells on a simple loop $Z^{+} \cap Z^{-}$such that $\operatorname{cl}\left(a_{1} \cup\right.$ $\left.a_{2} \cup \cdots \cup a_{m}\right)=Z^{+} \cap Z^{-}$, where $c l(X)$ means the closure of $X$. Let $A_{i}$ be the label of $a_{i}$, $1 \leq i \leq m$. Then we say that $\Lambda=A_{1} A_{2} \cdots A_{m}$ is a splitting cycle of $\Delta$ associated with $\left(Z^{+}, Z^{-}\right)$. We will call a splitting cycle $\Lambda=A_{1} A_{2} \cdots A_{m}$ an E-cycle if $A_{i} \neq A_{j}$ for each $i \neq j$.

Definition 2.3. Let $\sigma$ be a cell in $\Delta$. We say that $\sigma$ is positive if $\sigma \subset \operatorname{Int} Z^{+}$, negative if $\sigma \subset$ Int $Z^{-}$, and neutral if $\sigma \subset Z^{+} \cap Z^{-}$. We will use the notation $v(\alpha)$ for the number of neutral cells with label $\alpha$. 
It is easy to see the following.

Proposition 2.1. If $\alpha$ is a 2-label, then $v(\alpha)=0$. If $A$ is a 1-label, then $v(A)$ is either 1 or 3 . If $v$ is a 0 -label, then $2 \leq v(v) \leq 4$.

DEFINITION 2.4. A 1-label $A$ appearing in $\Delta$ is said to be bordered with respect to $\left(Z^{+}, Z^{-}\right)$if each of three 1-cells with the label $A$ is neutral. Otherwise, $A$ is said to be distributed with respect to $\left(Z^{+}, Z^{-}\right)$. Note that for a distributed 1-label $A$, there are three 1-cells with the 1-label $A$ such that one of them is positive, another is negative and the other is neutral.

We will introduce a new DS-deformation, named digging.

DEFINITION 2.5. Consider a DS-diagram $\Delta$ with a bicoloring $\left(Z^{+}, Z^{-}\right)$. Let $\sigma$ and $\tau$ be 1-cells in a connected component $R$ to $Z^{+}$. Suppose $A=f(\sigma)$ and $B=f(\tau)$, where $f$ is the identification map associated with a DS-diagram $\Delta$. Let $p \in \sigma$ and $q \in \tau$ be two points chosen so that $f(p) \neq f(q)$. Then there is a simple arc $\ell^{+}=\ell_{0}^{+} \cup \ell_{1}^{+} \cup \ell_{2}^{+} \cup \cdots \cup \ell_{m}^{+}$ transverse to 1-cells in $R$ such that

(1) each $\ell_{r}^{+}$is a directed arc with the initial point $p_{r-1}$ and the terminal point $p_{r}$, where $p_{0}=p$ and $p_{m}=q$,

(2) $\ell_{r}^{+} \cap \ell_{r+1}^{+}=p_{r}$,

(3) the interior Int $\ell_{r}^{+}$of $\ell_{r}^{+}$is in a positive 2-cell for each $r$, and $p_{r}$ is in a positive 1 -cell if $r \neq 0, m$,

(4) $f\left(p_{r}\right) \neq f(p)$ and $f(q)$ if $r \neq 0, m$.

We will call such a simple arc $\ell^{+}=\ell_{0}^{+} \cup \ell_{1}^{+} \cup \ell_{2}^{+} \cup \cdots \cup \ell_{m}^{+}$a mark-line joining $\sigma$ with $\tau$. Note that, for each simple arc $\ell_{r}^{+}$, there is the spouse $\ell_{r}^{-}$of $\ell_{r}^{+}$so that $f\left(\ell_{r}^{+}\right)=f\left(\ell_{r}^{-}\right)$and $\ell_{r}^{-} \cap\left(\ell_{0}^{+} \cup \ell_{1}^{+} \cup \ell_{2}^{+} \cup \cdots \cup \ell_{m}^{+}\right)=\emptyset$.

See Figure 3. Figure 3-c is obtained from Figure 3-a via Figure 3-b. We will say $d\left(\ell^{+}\right)$: Figure $3-\mathrm{a} \Rightarrow$ Figure $3-\mathrm{c}$ is a digging along a mark-line $\ell^{+}$, or simply a digging if there is no confusion.

\section{THEOREM 2.2. A digging is a DS-deformation.}

Proof. Suppose $d\left(\ell^{+}\right)$is a digging along a mark-line $\ell^{+}=\ell_{0}^{+} \cup \ell_{1}^{+} \cup \ell_{2}^{+} \cup \cdots \cup \ell_{m}^{+}$. If $m=0, d\left(\ell^{+}\right)=d\left(\ell_{0}^{+}\right)$is nothing but a piping, and hence $d\left(\ell^{+}\right)$is a DS-deformation. If $m=1, d\left(\ell^{+}\right)$is established by applying pipings twice. In general, $d\left(\ell^{+}\right)$is a consequence of $m+1$ times of applications of piping.

Suppose $\left\{R_{1}, R_{2}, \cdots, R_{p}\right\}$ and $\left\{S_{1}, S_{2}, \cdots, S q\right\}$ are the set of connected components of $Z^{+}$and $Z^{-}$. We will call $R_{i}$ a positive region and $S_{j}$ a negative region. In this situation, we will denote $\rho\left(Z^{+}, Z^{-}\right)=p+q$. Note that regions $R_{i}$ and $S_{j}$ are 2-disks with or without holes since a DS-diagram $\Delta$ is a diagram on a 2-sphere $S^{2}$ associated with a 3-regular connected graph. Hence at least one element of $\left\{R_{i}\right\} \cup\left\{S_{j}\right\}$ is a 2-disk. Without loss of generality we may assume $S_{q}$ is a 2-disk. Suppose $R_{p}$ is the positive region adjacent to $S_{q}$. If $p+q \geq 3$, there is another negtive region, say $S_{q-1}$, adjacent to $R_{p}$. 
REMODELING A DS-DIAGRAM
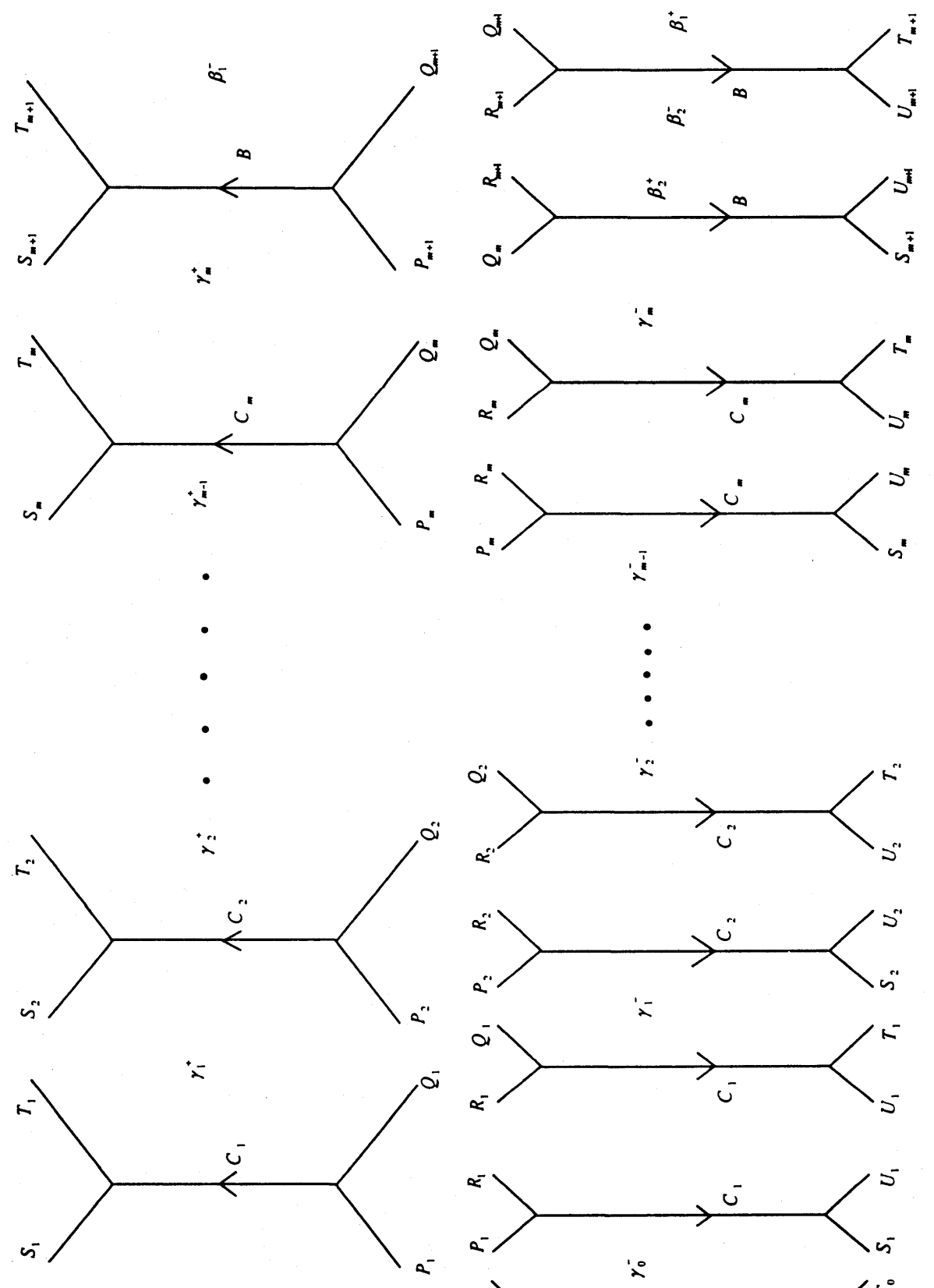

2
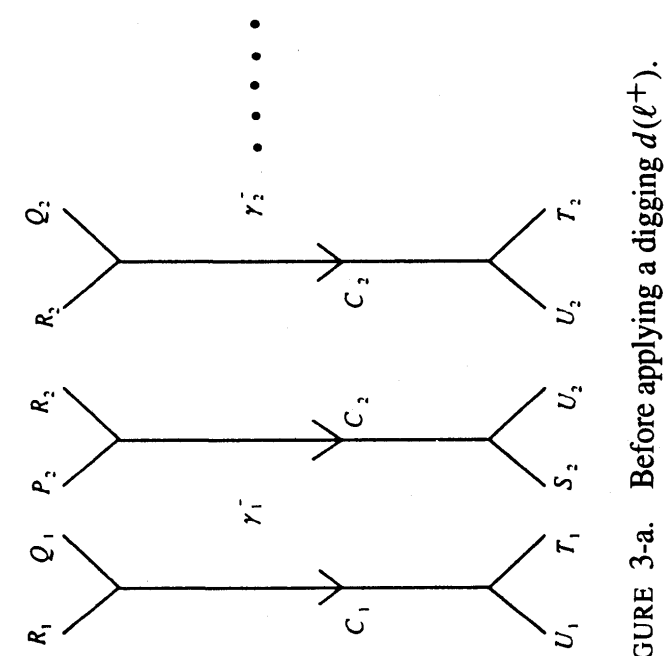

ஸे
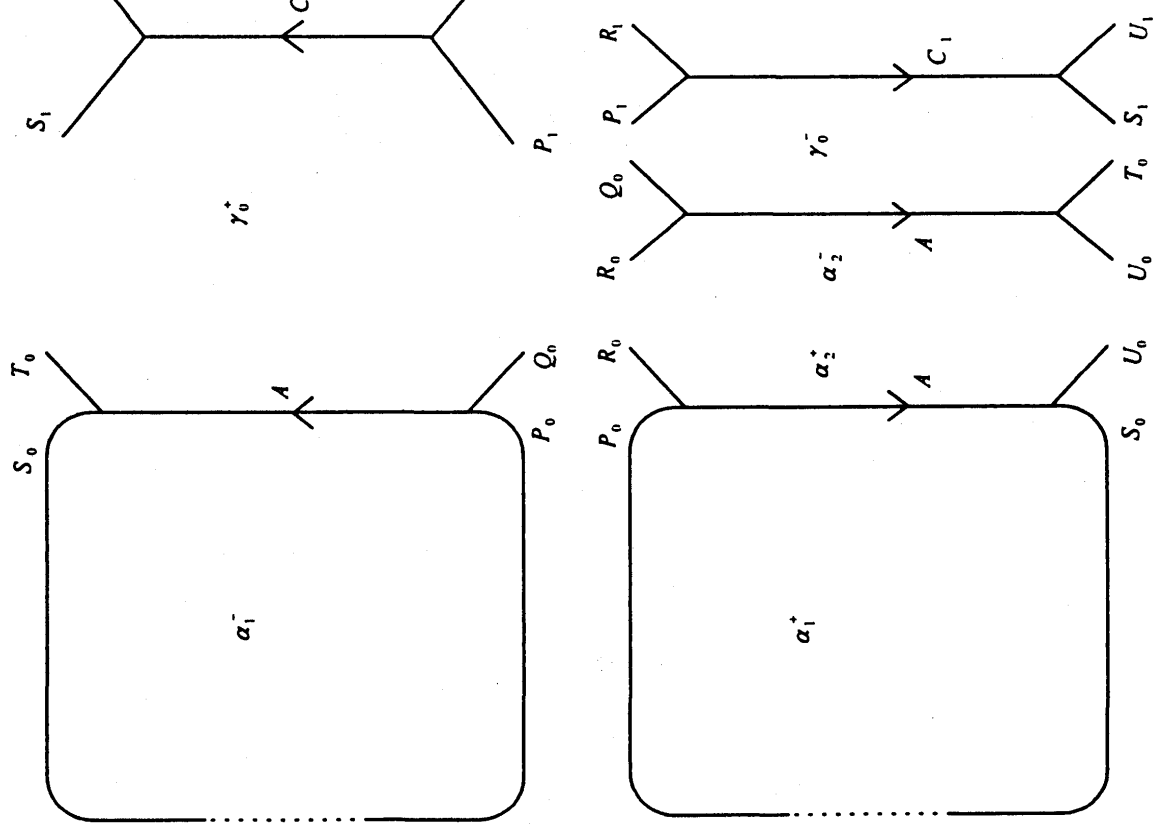

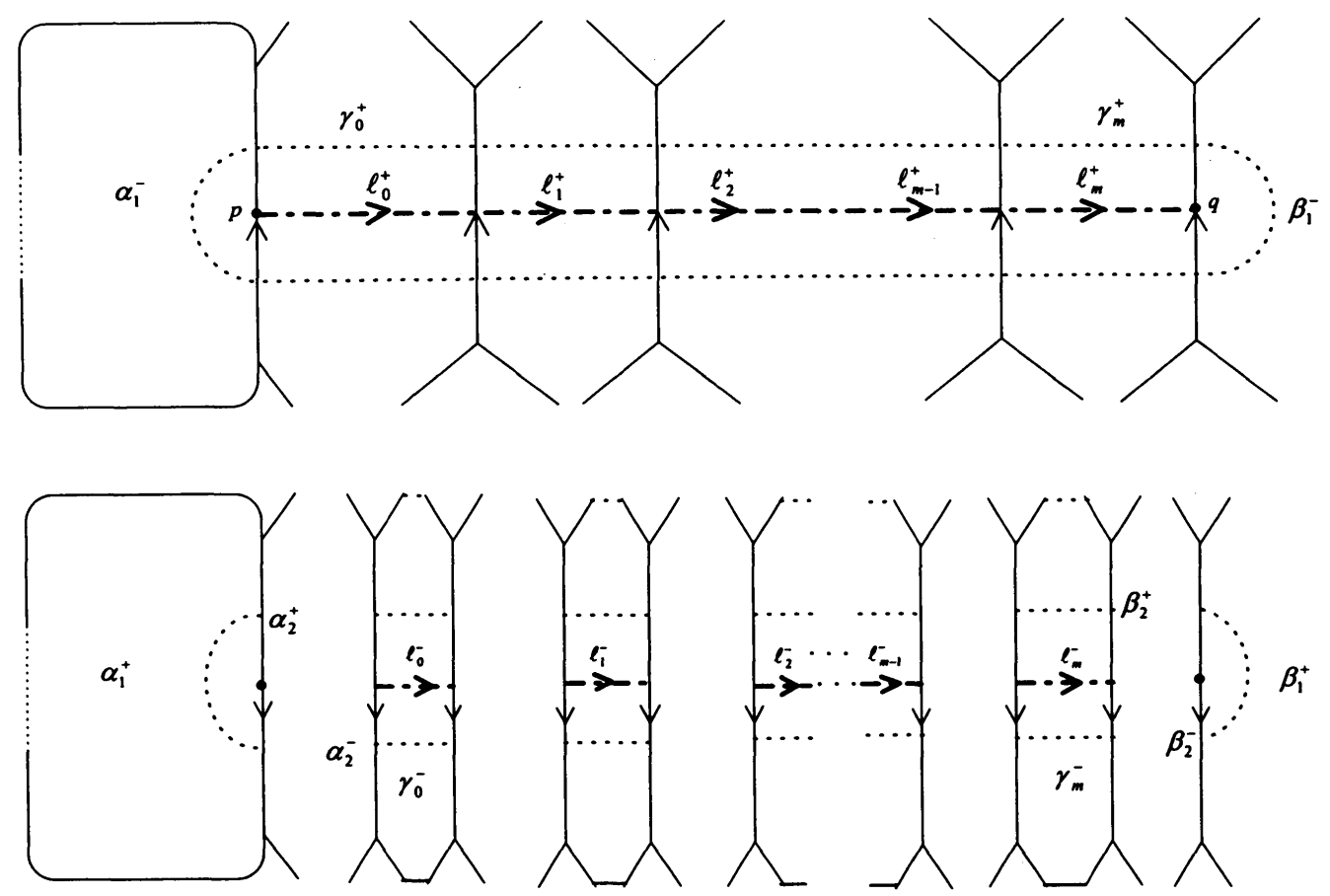

FIgURE 3-b. Mark line $\ell^{+}$.
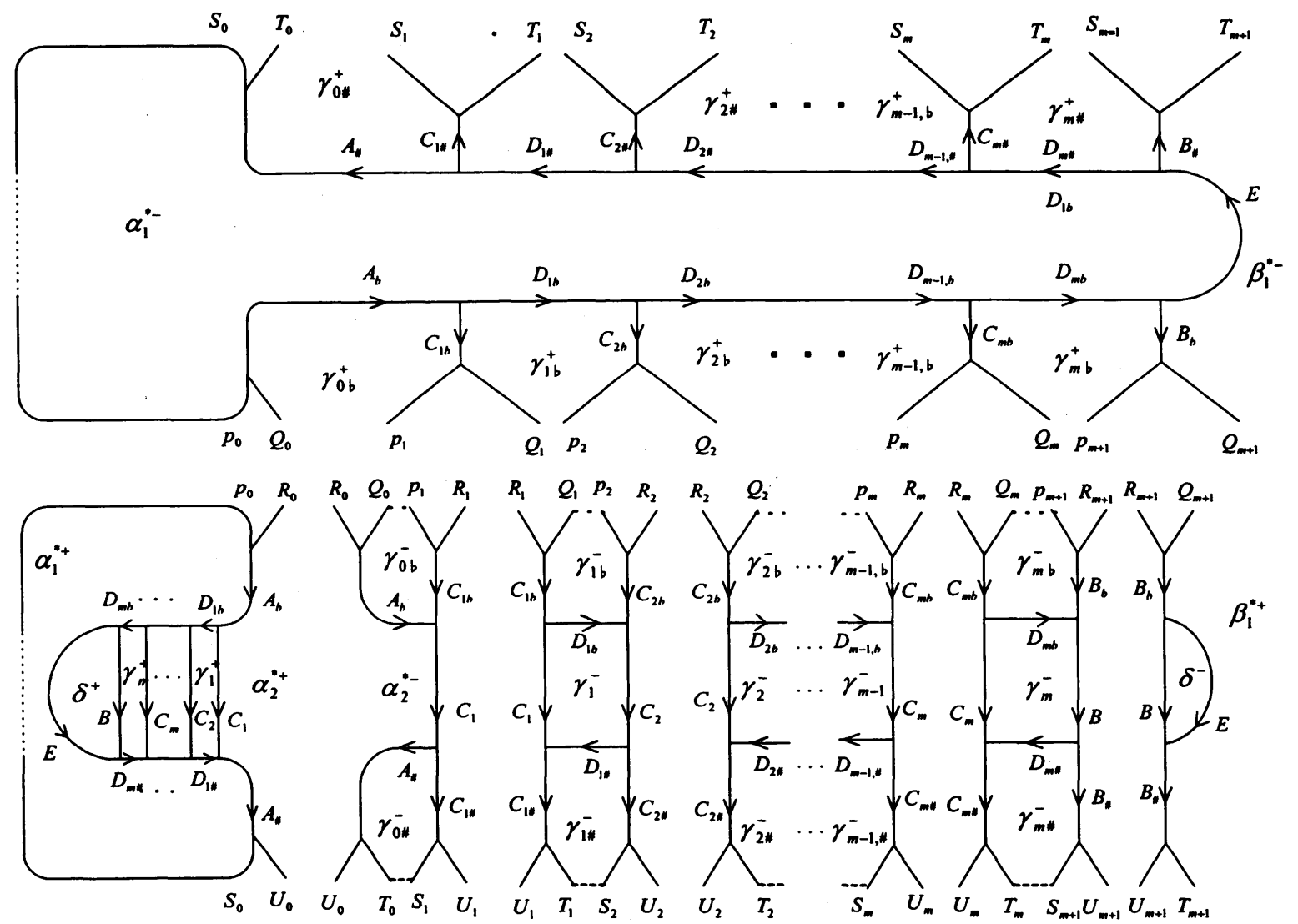

FIGURE 3-c. After applying a digging $d\left(\ell^{+}\right)$. 
LEMMA 2.1. Suppose $p+q \geq 3$. Let $\sigma \subset R_{p} \cap S_{q}$ and $\tau \subset R_{p} \cap S_{q-1}$ be neutral 1-cells. Suppose the label of $\sigma$ is distributed and the label of $\tau$ is bordered. Then there is a DS-diagram $\Delta^{\prime}$ with a bicoloring $\left(Z^{\prime+}, Z^{\prime-}\right)$ such that $\Delta^{\prime}$ is DS-isomorphic to $\Delta$ and $\rho\left(Z^{\prime+}, Z^{\prime-}\right)=p+q-1$.

Proof. Applying a digging along a proper arc in $R_{p}$ joining 1-cells $\sigma$ and $\tau$, we will be able to obtain a required DS-diagram $\Delta^{\prime}$ as follows. Let $A$ and $B$ be 1-labels of $\sigma$ and $\tau$. Suppose $\alpha_{1}^{ \pm}, \alpha_{2}^{ \pm}, \beta_{1}^{ \pm}, \beta_{2}^{ \pm}, \gamma_{r}^{ \pm}(0 \leq r \leq m)$ are 2-cells in $\Delta$ as shown in Figure 3-a where $\alpha_{1}^{-} \subset S_{q}, \gamma_{0}^{+} \cup \gamma_{1}^{+} \cup \cdots \cup \gamma_{m}^{+} \subset R_{p}, \beta_{1}^{-} \subset S_{q-1}$. Let $\ell^{+}$be the closure of $\ell_{0}^{+} \cup \ell_{1}^{+} \cup \ell_{2}^{+} \cdots \cup \ell_{m}^{+}$ which is a proper arc in the closure of $\gamma_{0}^{+} \cup \gamma_{1}^{+} \cup \cdots \cup \gamma_{m}^{+}$joining a point $p \in \sigma$ to a point $q \in \tau$ (see Figure 3-b). By a digging $d\left(\ell^{+}\right)$along $\ell^{+}, \alpha_{i}$ and $\beta_{i}(i=1,2)$ are replaced by $\alpha_{i}^{*}$ and $\beta_{i}^{*}$, and $\gamma_{i}$ is replaced by two 2-labels $\gamma_{i \sharp}$ and $\gamma_{i \text { b }}$ and further, a new 2-label $\delta$ with $\partial \delta=B E^{-1}$ is born. Note that $\gamma_{0 \sharp}^{+} \cup \gamma_{1 \sharp}^{+} \cup \cdots \cup \gamma_{m \sharp}^{+}$and $\gamma_{0 b}^{+} \cup \gamma_{1 b}^{+} \cup \cdots \cup \gamma_{m b}^{+}$are in the same region, say $R_{p}^{\prime}$, since $S_{q}$ is a 2-cell. We can see the resulting DS-diagram $\Delta^{\prime}$ (Figure 3-c) has a natural bicoloring $\left(Z^{\prime+}, Z^{\prime-}\right)$ such that $\rho\left(Z^{\prime+}, Z^{\prime-}\right)=p+q-1$.

THEOREM 2.3. See Figure 4. Suppose $\Delta_{1}$ is a DS-diagram with a symbolic representation, see [6],

$$
\{\cdots B \cdots, \cdots B \cdots, \cdots B \cdots, \cdots \cdots\},
$$

where $B$ is a distributed 1-label. Suppose $\Delta_{2}$ is a DS-diagram with a symbolic representation

$$
\left\{\partial \zeta, \partial \eta, \partial \lambda, \partial \mu, \partial \nu, \partial \gamma_{m}, \partial \beta_{1}, \partial \beta_{2}, \cdots \cdots\right\},
$$

where

$$
\begin{aligned}
& \partial \zeta=B_{*} P_{1} P_{2} B_{*} U V, \quad \partial \eta=B_{*} Q_{1} Q_{2}, \\
& \partial \lambda=T^{-1} S^{-1}, \quad \partial \mu=P_{2}^{-1} T Q_{2}, \quad \partial \nu=Q_{1} S P_{1}^{-1}, \\
& \partial \gamma_{m}=\cdots B_{1} V Q_{2}^{-1} S P_{2} V^{-1} B_{2} \cdots, \\
& \partial \beta_{1}=\cdots B_{1} U^{-1} P_{1} T Q_{1}^{-1} U B_{2} \cdots, \\
& \partial \beta_{2}=\cdots B_{2}^{-1} B_{1}^{-1} \cdots .
\end{aligned}
$$

Then $\Delta_{1}$ and $\Delta_{2}$ are DS-isomorphic to each other.

Proof. Applying $\mathrm{II}^{-}$-deformation $\Psi^{-1}(\eta)$ to $\Delta_{2}$, we obtain a DS-diagram $\Delta_{3}$ with a symbolic representation:

$$
\begin{aligned}
\left\{P_{1} P_{2} U V, T^{-1} S^{-1} W_{*}^{-1}, T P_{2}^{-1}, S P_{1}^{-1} ;\right. & \cdots B_{1} V S P_{2} W_{*} V^{-1} B_{2} \cdots, \\
& \left.\cdots B_{1} U^{-1} W_{*} P_{1} T U B_{2} \cdots, \cdots B_{2}^{-1} B_{1}^{-1} \cdots\right\} .
\end{aligned}
$$

Then applying 2-gon collapsing $\delta\left(S P_{1}^{-1}\right)$ to $\Delta_{3}$, we obtain a DS-diagram $\Delta_{4}$ with a symbolic representation:

$$
\left\{W_{* *}^{-1}, U V_{*} ; \cdots B_{1} V_{*} W_{* *} V_{*}^{-1} B_{2} \cdots, \cdots B_{1} U^{-1} W_{* *} U B_{2} \cdots, \cdots B_{2}^{-1} B_{1}^{-1} \cdots\right\} .
$$

Again applying 2-gon collapsing $\delta\left(U V_{*}\right)$ to $\Delta_{4}$, we obtain $\Delta_{1}$. Hence $\Delta_{1}$ and $\Delta_{2}$ are DS-isomorphic to each other. 


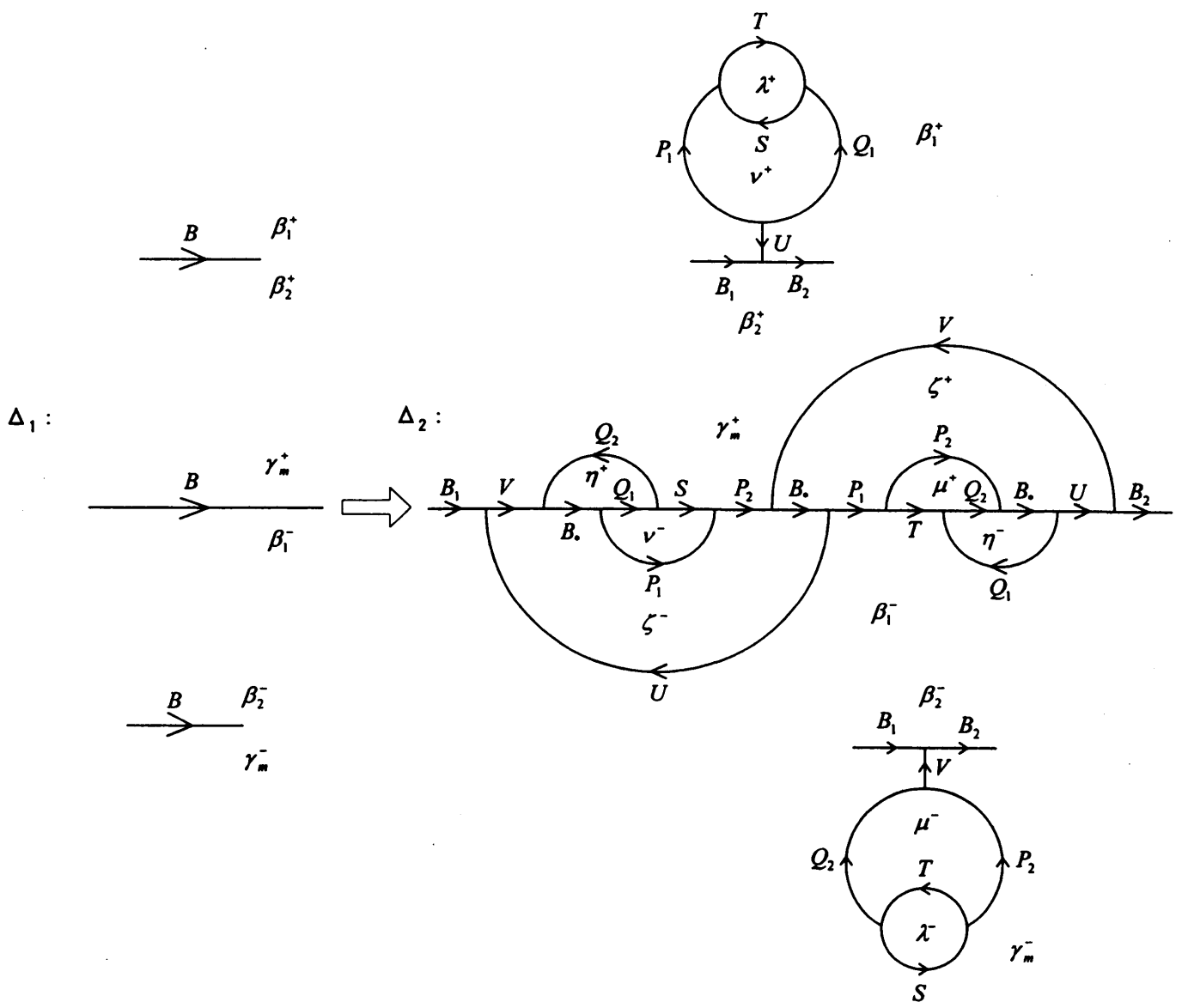

FIGURE 4. Deformation to replacing a distributed 1-label by a bordered one.

ProOF OF THEOREM 2.1. We will establish our proof by the induction on $n=$ $\rho\left(Z^{+}, Z^{-}\right)$. If $n=2$, then $\Delta$ is already a splittable DS-diagram. Hence we assume $n \geq 3$. And we show that there is a DS-diagram $\Delta^{\prime}$ with a bicoloring $\left(Z^{\prime+}, Z^{\prime-}\right)$ such that $\Delta^{\prime}$ is DS-siomorphic to $\Delta$ and $\rho\left(Z^{\prime+}, Z^{\prime-}\right)=n-1$. It is enough to consider the situation that

(1) $\left\{R_{1}, R_{2}, \cdots, R_{p}\right\}$ and $\left\{S_{1}, S_{2}, \cdots, S_{q}\right\}$ are connected components of $Z^{+}$and $Z^{-}$, and $p+q=n$,

(2) $S_{q}$ is a 2-disk, and $R_{p}$ is the positive region which is adjacent to both of $S_{q}$ and $S_{q-1}$

We will attempt to replace $S_{q}$ and $S_{q-1}$ by a new negative region $S_{q-1}^{\prime}$ through DS-deformation on $\Delta$.

Step 1. In this step, we show that we can assume there is a neutral 1-cell in $S_{q} \cap R_{p}$ with a distributed 1-label $A$. If there is no such 1-cell in $S_{q} \cap R_{p}$, we claim that we can change $\Delta$ to $\Delta_{*}$ with a bicoloring $\left(Z_{*}^{+}, Z_{*}^{-}\right)$having the regions $\left\{R_{* 1}, R_{* 2}, \cdots, R_{* p}\right\}$ and $\left\{S_{* 1}, S_{* 2}, \cdots, S_{* q}\right\}$ such that

(1) $S_{* q}$ is a 2-disk and $R_{* p}$ is adjacent to $S_{* q}$, 


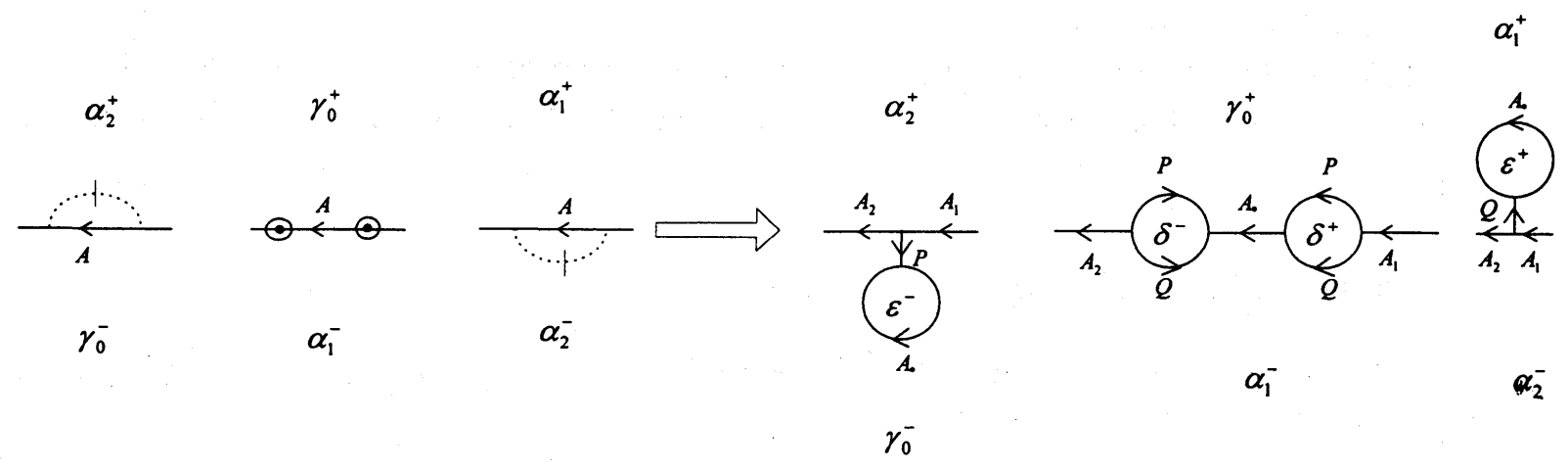

FIGURE 5. Deformation replacing a bordered 1-label by a distributed one.

(2) $S_{* q} \cap R_{* p}$ contains a neutral 1-cell with a distributed 1-label, say $A_{*}$.

We can construct $\Delta_{*}$ as follows. Choose one of the 1-cells, say $\sigma$, in $S_{q} \cap R_{p}$ and suppose the 1-label $A=f(\sigma)$ of $\sigma$ is bordered. Let $\alpha_{1}, \alpha_{2}, \gamma_{0}$ be 2-labels of $\Delta$ such that $A \subset$ $\partial \alpha_{1}, \partial \alpha_{2}, \partial \gamma_{0}$. Some of $\alpha_{1}, \alpha_{2}, \gamma_{0}$ may possibly be coincide together. Since $A$ is bordered, we obtain the left of Figure 5, especially we may assume $\alpha_{1}^{-} \subset S_{q}$.

Suppose $\ell$ is a proper arc in $\alpha_{2}$ joining two points on $A$. Carrying out the piping $L_{0}(A)$ : $\Delta \rightarrow \Delta_{*}$ along $\ell$, we obtain

$$
\Delta_{*}=\left\{A_{*}, P Q^{-1} ; A_{1}^{+} P A_{*} P^{-1} A_{2}^{-}, A_{1}^{+} Q A_{*} Q^{-1} A_{2}^{-}, A_{1}^{+} A_{2}^{-}\right\}
$$

which is DS-isomorphic to $\Delta$. This DS-diagram $\Delta_{*}$ has a natural bicoloring $\left(Z_{*}^{+}, Z_{*}^{-}\right)$such that $\rho\left(Z_{*}^{+}, Z_{*}^{-}\right)=p+q$ and $\alpha_{1}^{-} \cup \delta^{-} \subset S_{q}$, where $\delta^{-}$is a 2-cell with 2-label $\delta$ so that $\partial \delta=P Q^{-1}$. A 1-label $A_{*}$ is a distributed one on a new $S_{* q} \cap R_{* p}$ with respect to $\left(Z_{*}^{+}, Z_{*}^{-}\right)$.

Step 2. Suppose $\Delta$ is a DS-diagram with a bicoloring $\left(Z^{+}, Z^{-}\right)$having the regions $\left\{R_{1}, R_{2}, \cdots, R_{p}\right\}$ and $\left\{S_{1}, S_{2}, \cdots, S_{q}\right\}, p+q=n$, such that

(1) $S_{q}$ is a 2-disk and $R_{p}$ is adjacent to $S_{q}$,

(2) $S_{q} \cap R_{p}$ contains a neutral 1-cell with a distributed 1-label, and

(3) $S_{q-1}$ is adjacent to $R_{p}$.

We want to find a neutral 1-cell in $R_{p} \cap S_{q-1}$ with bordered 1-label. Suppose there is no such 1-cell in $R_{p} \cap S_{q-1}$. Choose an arbitrary 1-cell, say $\tau$, in $R_{p} \cap S_{q-1}$. Then the 1label $B=f(\tau)$ of $\tau$ does not appear in $S_{q} \cap R_{p}$ since $B$ is distributed and $\tau \subset R_{p} \cap S_{q-1}$ is neutral. Applying the DS-deformation in Theorem 2.3 to the 1-label $B$, we obtain the required DS-diagram $\Delta_{*}$ and a new 1-label $B_{*}$.

Step 3. From the argument of Step 1 and Step 2, if necessary, we can seek for a DS-diagram $\Delta_{*}$ with a bicoloring $\left(Z_{*}^{+}, Z_{*}^{-}\right)$having the regions $\left\{R_{* 1}, R_{* 2}, \cdots, R_{* p}\right\}$ and $\left\{S_{* 1}, S_{* 2}, \cdots, S_{* q}\right\}, p+q=n$, such that

(1) $S_{* q}$ is a 2-disk and $R_{* p}$ is adjacent to $S_{* q}$,

(2) $S_{* q} \cap R_{* p}$ contains a neutral 1-cell $\sigma_{*}$ with a distributed 1-label,

(3) $S_{*, q-1}$ is adjacent to $R_{p}$, 
(4) $R_{* p} \cap S_{*, q-1}$ contains a neutral 1-cell $\tau_{*}$ with a bordered 1-label,

(5) $\Delta_{*}$ is DS-isomorphic to $\Delta$.

To $\Delta_{*}$ applying the digging along a proper arc in $R_{* p}$ joining $\sigma_{*}$ and $\tau_{*}$, we will obtain a DS-diagram $\Delta^{\prime}$ with a bicoloring $\left(Z^{\prime+}, Z^{\prime-}\right)$ such that $\rho\left(Z^{\prime+}, Z^{\prime-}\right)=n-1$. This completes the proof.

\section{Remodeling a splittable DS-diagram.}

In this section, let $\Delta$ be a splittable DS-diagram with $\left(Z^{+}, Z^{-}\right)$, and $\Lambda$ a splitting cycle of $\Delta$ associated with $\left(Z^{+}, Z^{-}\right)$.

Proposition 3.1. Suppose $A$ is an arbitrary 1-label of $\Delta$. Then $A$ appears on $\Lambda$ exactly once if $A$ is distributed, and exactly three if $A$ is bordered.

Proof. It is an obvious since a 1-cell $\sigma$ with 1-label $A$ is contained in $Z^{+} \cap Z^{-}$if and only if $\sigma$ is neutral.

PROPOSITION 3.2. If $\Lambda=\cdots A A \cdots$, then we have $\Lambda=\cdots A A A \cdots$.

PROoF. It is easy to see that a DS-diagram contains a path $A A A$ if it contains a path $A A$. And further, $A$ is bordered since $A$ appears on $\Lambda$ at least twice as $A A$. Therefore the third edge with 1-label $A$ is also contained in $\Lambda$.

Definition 3.1. A splitting cycle $\Lambda=A_{1} A_{2} \cdots A_{m}$ is called a good cycle if $A_{i} \neq$ $A_{i+1}$ for each $i(\bmod m)$.

LEMMA 3.1. There is a splittable DS-diagram $\Delta_{*}$ with a good cycle such that $\Delta_{*}$ is DS-isomorphic to $\Delta$.

Proof. If a splitting cycle $\Lambda$ does not contain any adjacent 1-cells with the same 1-label, $\Lambda$ is already a good cycle. Suppose not. Then there is a 1-label $A$ so that $\Lambda=$ $w_{1} A A A w_{2}$. In this case, there are two kind of surroundings of $A$ (Figure 6-a):

$$
\begin{aligned}
& \Sigma_{1}(A)=\left\{X^{+} A A Y^{-}, Y^{-} A X^{+}, Y^{-} X^{+}\right\}, \quad \text { and } \\
& \Sigma_{2}(A)=\left\{Y^{-} A A X^{+}, X^{+} A Y^{-}, Y^{-} X^{+}\right\} .
\end{aligned}
$$

Note that $X^{ \pm} \neq A$ and $Y^{ \pm} \neq A$, but possibly $Y=X^{ \pm}$. For each case, we claim that we can obtain a DS-diagram $\Delta_{*}$ with a splitting cycle $\Lambda_{*}=w_{1} X A P R A Q S A Y w_{2}$. For $\Sigma_{1}(A)$, if we apply an elementary deformation $\Phi=\Phi\left(X^{+} A^{-}, A^{+} Y^{-}\right)$of type $I^{+}$, we obtain

$$
\Phi\left(\Sigma_{1}(A)\right)=\left\{P Q^{-1}, R S^{-1}, X^{+} A Q R A Y^{-}, Y^{-} Q S A P R X^{+}, Y^{-} P S X^{+}\right\},
$$

(see Figure 6-b). It is similar to $\Sigma_{2}(A)$. By repeating this operation, we can obtain a splittable DS-diagram $\Delta_{*}$ with a good cycle.

As we saw in $\S 2$, any DS-diagram is DS-isomorphic to a splittable one with a splitting cycle $\Lambda$. Furthermore, by the previous lemma, we may assume $\Lambda$ is a good cycle, that is, $\Lambda$ does not contain $A A$, where $A$ is a 1-label. We will start with this situation. Note that $\Lambda$ consists of distributed 1-labels (each of them appears exactly once on $\Lambda$ ) and arc-type 

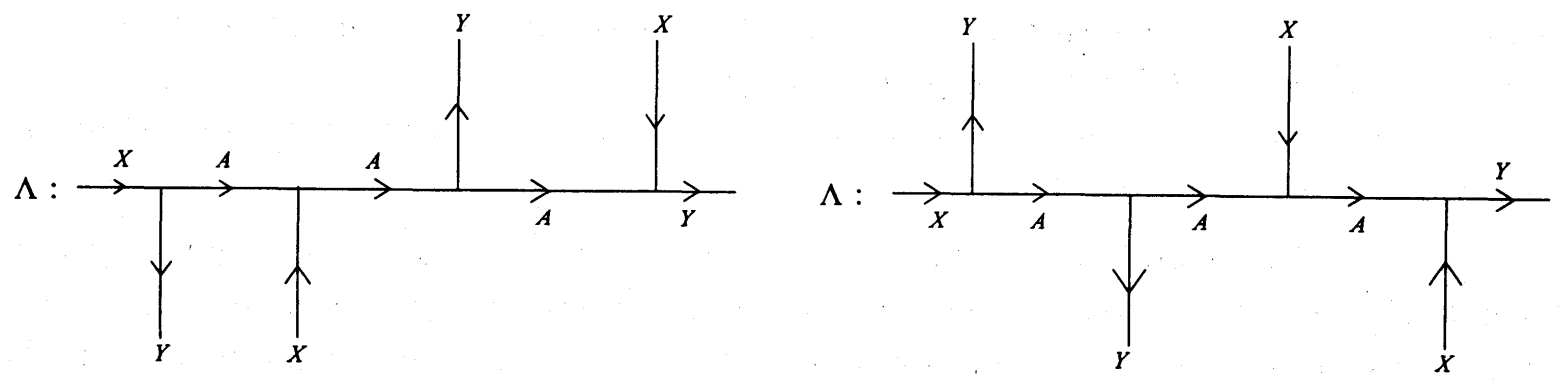

FIGURE 6-a. Surroundings of a 1-label $A$ with $A A$ on $\Lambda$.

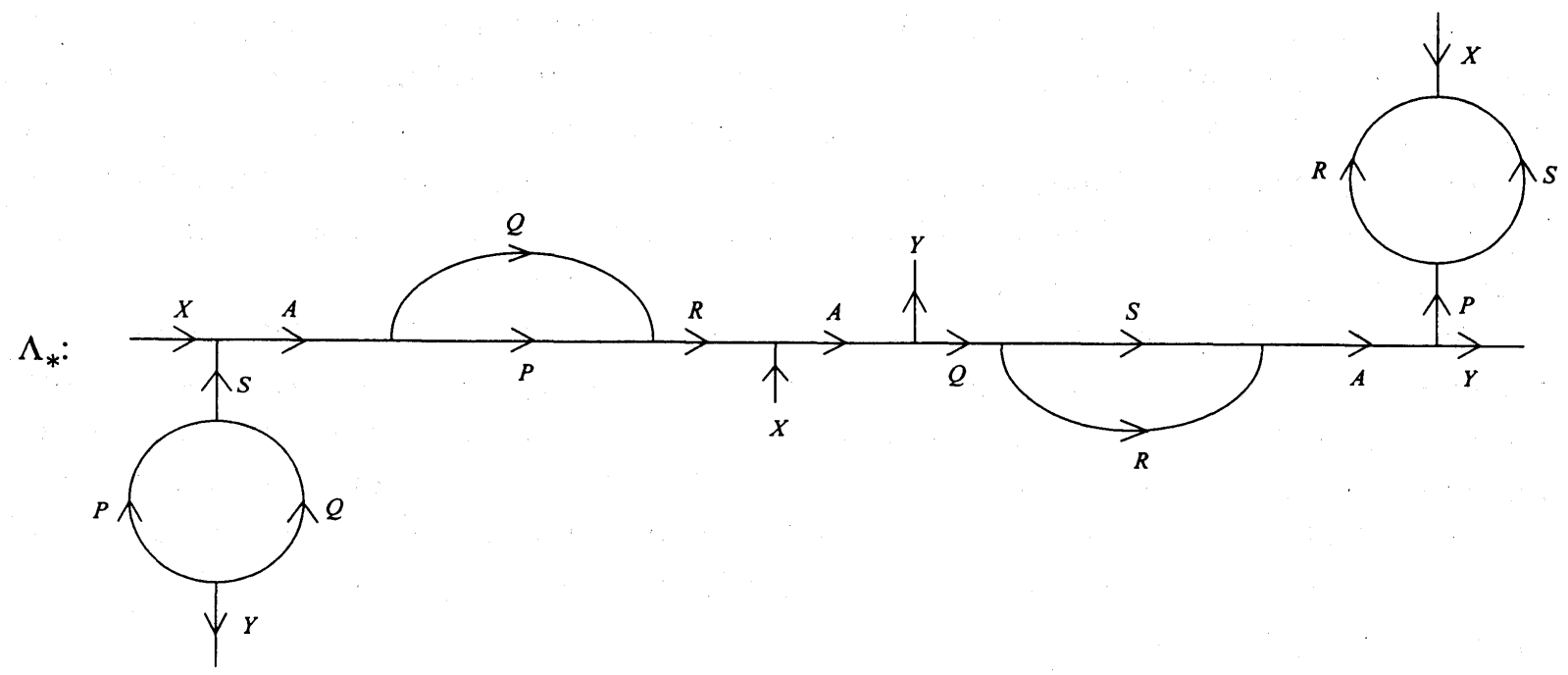

FIGURE 6-b. $\Phi\left(\Sigma_{1}(A)\right)$.

bordered 1-labels (each of them appears just three times on $\Lambda$ ), see [6]. We will eliminate these bordered 1-labels from $\Lambda$ step by step. Then, a resulting splitting cycle $\Lambda_{*}$ will not contain any bordered 1-label, and hence $\Lambda_{*}$ will be automatically an E-cycle of a DS-diagram which is DS-isomorphic to the original DS-diagram.

PROPOSITION 3.3. Suppose $\tau$ is a neutral 0 -cell in $\Delta$ and $\sigma_{1}, \sigma_{2}, \sigma_{3}$ are 1-cells incident to $\tau$. Then either $\sigma_{1}, \sigma_{2}, \sigma_{3} \in Z^{+}$or $\sigma_{1}, \sigma_{2}, \sigma_{3} \in Z^{-}$holds; $\tau$ is said to be positive handed if $\sigma_{1}, \sigma_{2}, \sigma_{3} \in Z^{+}$, and negative handed otherwise.

PROOF. Since $\tau$ is neutral, $\tau \subset Z^{+} \cap Z^{-}$, and two of $\sigma_{1}, \sigma_{2}, \sigma_{3}$ are also on $Z^{+} \cap Z^{-}$. Hence either $\sigma_{1}, \sigma_{2}, \sigma_{3} \in Z^{+}$or $\sigma_{1}, \sigma_{2}, \sigma_{3} \in Z^{-}$holds.

Remember $v(\alpha)$ means the number of the neutral cells with the label $\alpha$.

Proposition 3.4. Let $v$ be a 0 -label of $\Delta$. Then $2 \leq v(v) \leq 4$.

(1) Suppose $v(v)=2$. Then one of the neutral 0-cells with label $v$ is positive handed and the other is negative handed. 
(2) Suppose $v(v)=3$. Then three neutral 0-cells with label $v$ are either positive handed all or negative handed all. If these neutral 0 -cells are positive (negative) handed, the non-neutral 0-cells with label $v$ is negative (positive).

(3) Suppose $v(v)=4$. Then two of the 0 -cells with label $v$ are positive handed and the other two are negative handed.

PROOF. For an arbitrary 0-label $v$ in $\Delta$, there are four 0-cells with the same 0-label $v$. Each 0-cell has three 1-cells as hands, and has three corners, see [6]. Hence there are twelve angles corresponding to six corners for a 0-label $v$. Six of these angles are in $Z^{+}$and the others are in $Z^{-}$. This fact leads us to the proof.

Let $\Lambda$ be a good cycle. Let $A$ be a bordered 1-label with the initial 0-label $x$ and the terminal 0-label $y . x \neq y$ and that three 1-cells with label $A$ are all on the good cycle $\Lambda$. Let $\sigma$ be a 1-cell with label $A$ and $p, q$ be the initial and the terminal 0 -cell of $\sigma$; that is, $f(\sigma)=A, f(p)=x$ and $f(q)=y$. Then there are four types of $\sigma$ on $\Lambda$ (Figure 7). If $p$ and $q$ are both positive handed (negative handed), then we say $\sigma$ is of type $U^{+}$(type $U^{-}$). If $p$ is negative handed (positive handed) and $q$ is positive handed (negative handed), then we say $\sigma$ is of type $N^{+}$(type $N^{-}$, respectively).

We will consider patterns of the intersection of good cycle $\Lambda$ and surroundings around $\boldsymbol{A}$. Note that $3 \leq v(x), v(y) \leq 4$. Hence $(v(x), v(y))$ is one of $(3,3),(3,4),(4,3),(4,4)$. Note that $(3,4)$ and $(4,3)$ are essentially of the same type. Hence we get typical eight patterns (see Figure 8). They are denoted by $(v(x), v(y): N(A), U(A))$; where $N(A)$ is the number of 1-cells, with label $A$, of type $N^{+}$and $N^{-}$, and $U(A)$ the number of 1-cells with type $U^{+}$and $U^{-}$. The list is: $(3,3: 0,3),(3,3: 3,0),(4,3: 1,2),(4,3: 2,1),(4,4: 0,3),(4,4: 1,2)$, $(4,4: 2,1),(4,4: 3,0)$.

DEFINITION 3.2. A good cycle $\Lambda$ is called a better cycle if $(\nu(x), \nu(y): N(A), U(A))$ $=(3,3: 0,3)$ for each bordered 1-label $A$.

$b(\Delta)$ means the number of bordered 1-labels of $\Delta$.

THEOREM 3.1. Let $\Lambda$ be a good cycle and $b(\Delta)=k$. If there is a bordered 1-label whose pattern in not $(3,3: 0,3)$, we can reform $\Delta$ to a DS-diagram $\Delta_{*}$ with a good cycle and $b\left(\Delta_{*}\right)=k-1$.

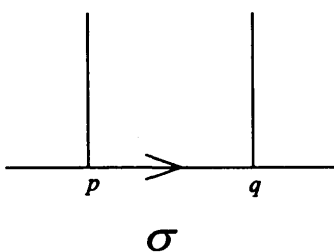

Type $U^{+}$

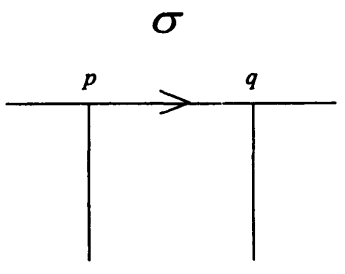

Type $U^{-}$

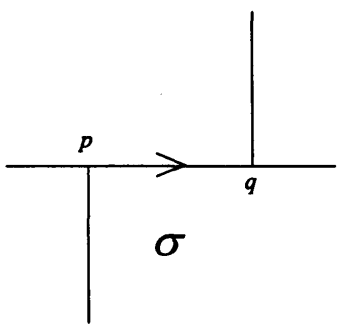

Type $N^{+}$

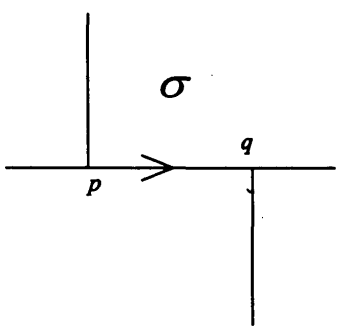

Type $N^{-}$

FIgURE 7. Type of 1-cell on $\Lambda$. 
$\Lambda:$
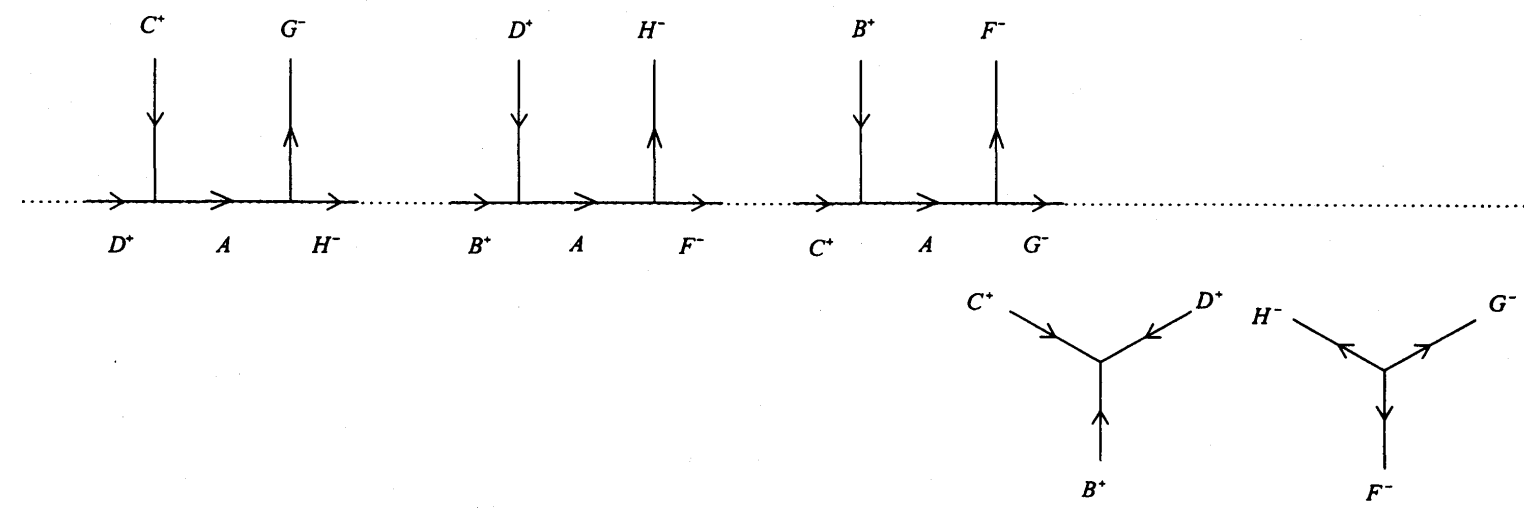

Pattern 0: $(3,3: 0,3)$.

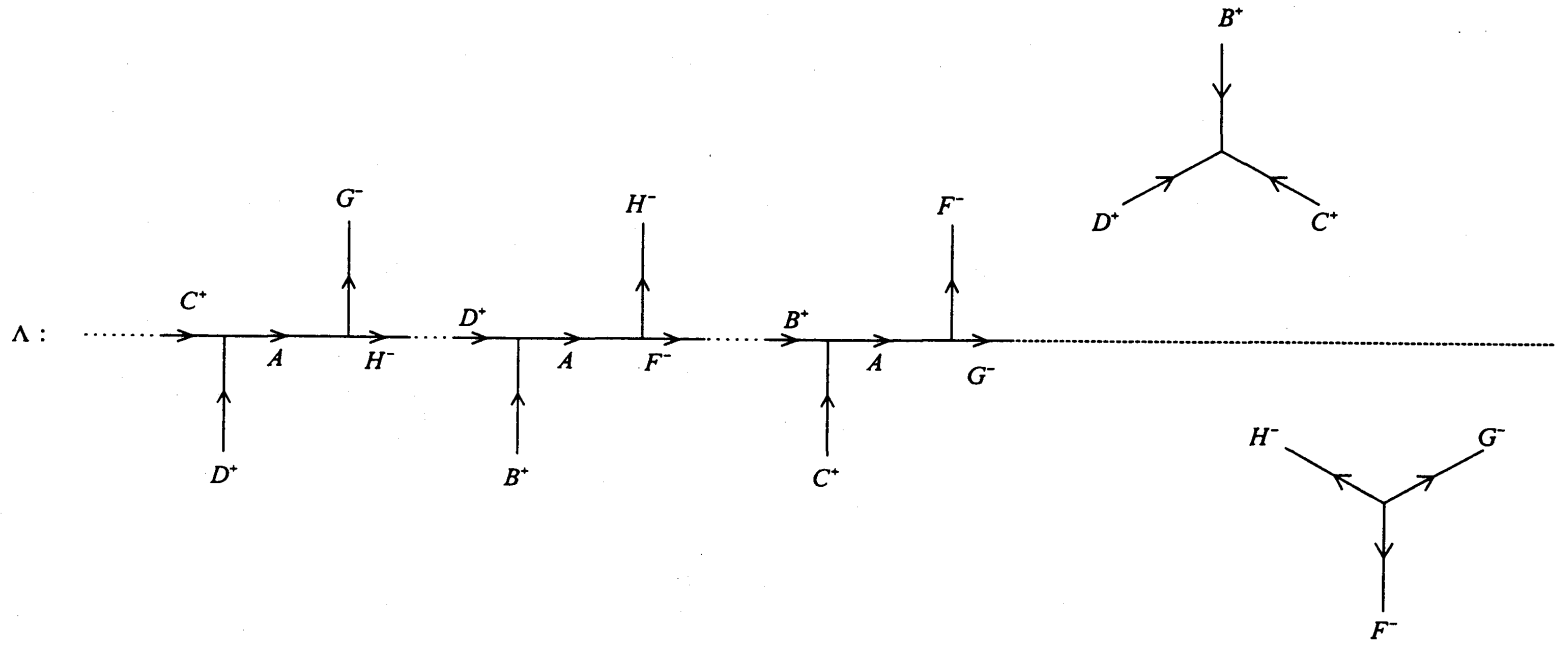

Pattern 1: $(3,3: 3,0)$.

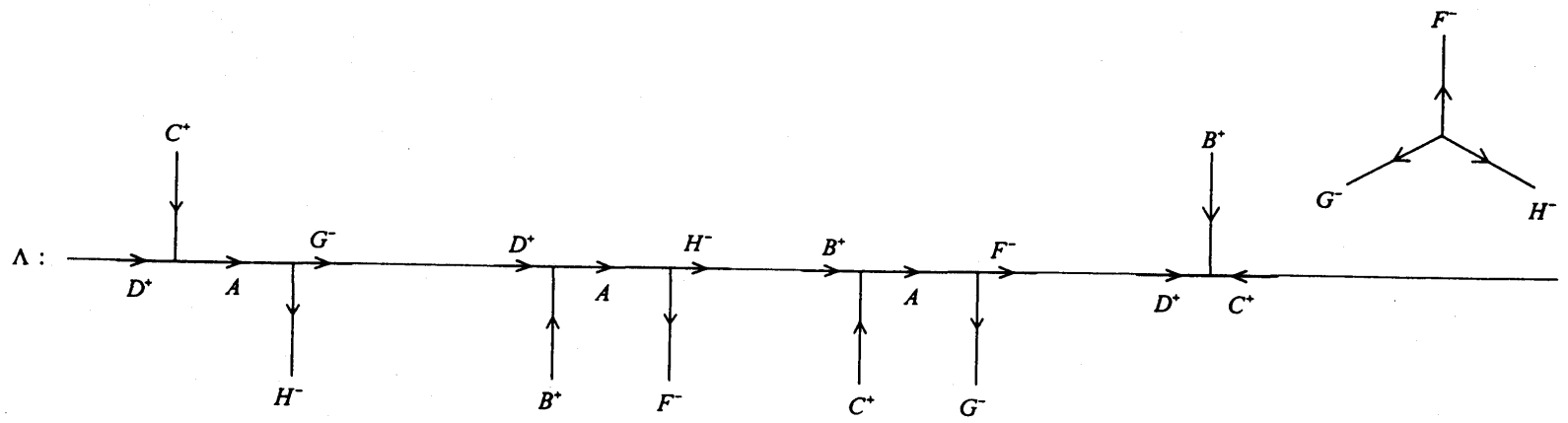

Pattern 2: $(4,3: 1,2)$. 
$\Lambda:$

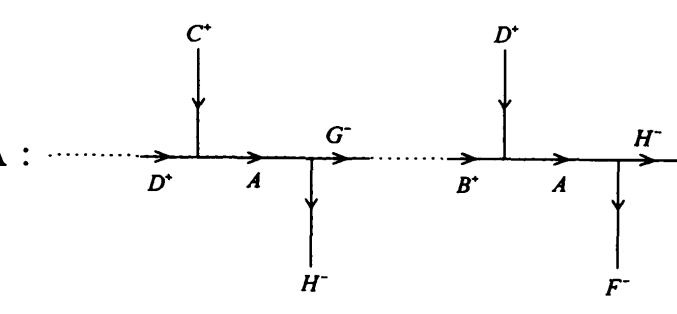
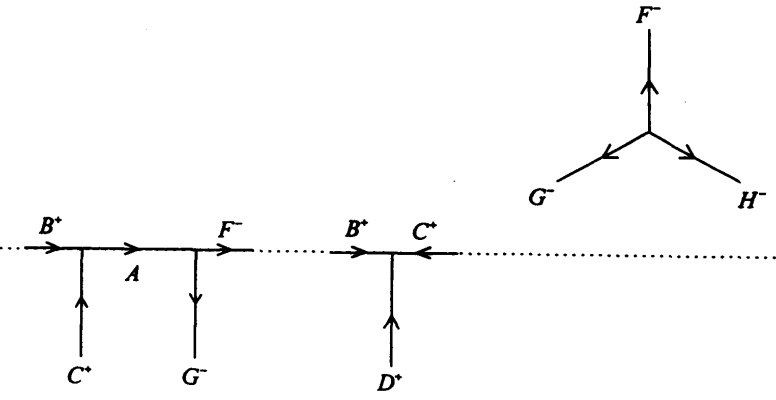

Pattern 3: $(4,3: 2,1)$.

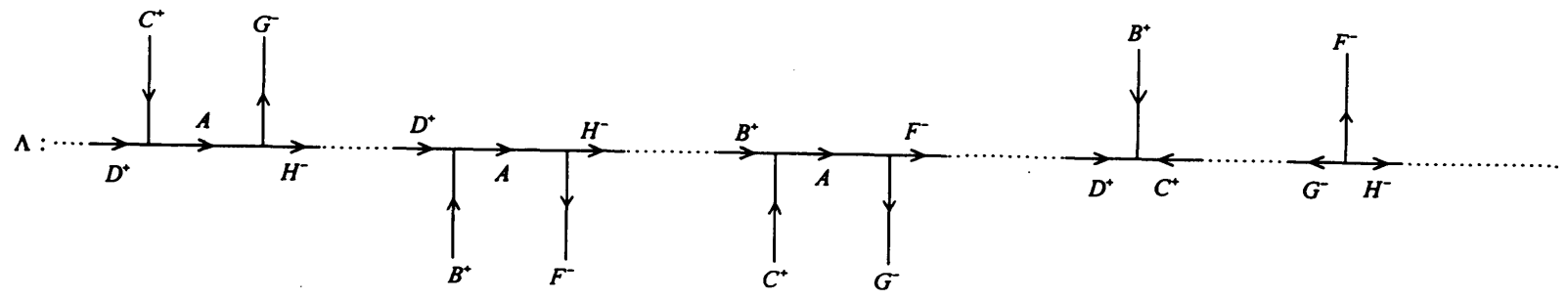

Pattern 4: $(4,4: 0,3)$.

$\Lambda$ :

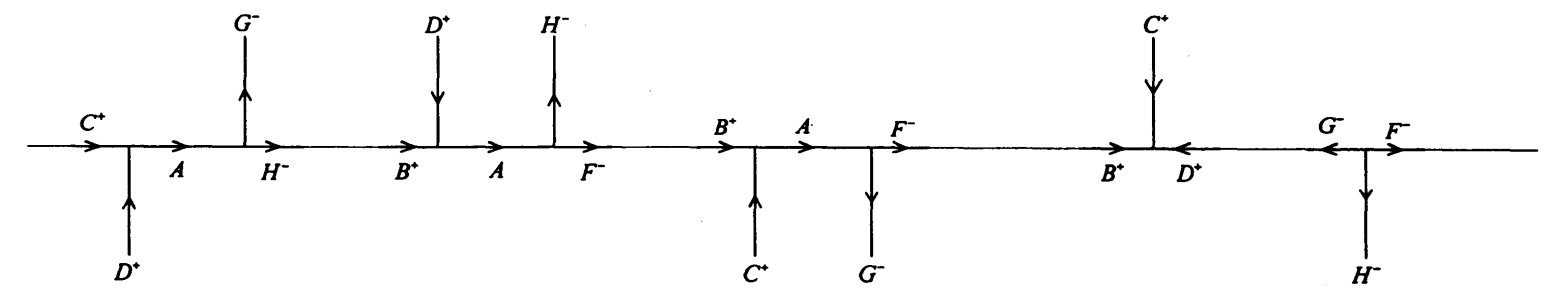

Pattern 5: $(4,4: 1,2)$.

$\Lambda$ :

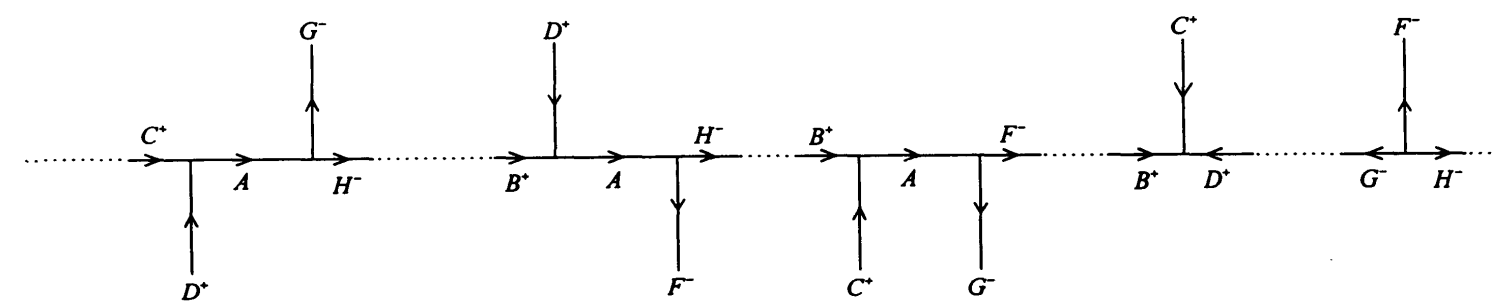

Pattern 6: $(4,4: 2,1)$.

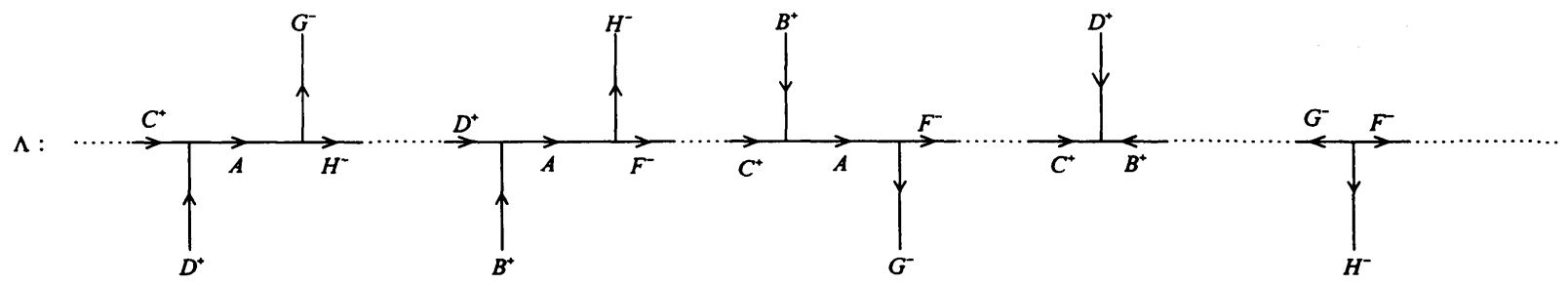

Pattern 7: $(4,4: 3,0)$.

FIGURE 8. Eight patterns of bordered 1-labels on a good cycle $\Lambda$. 
Proof. Suppose $A$ is a bordered 1-label whose pattern is not $(3,3: 0,3)$. For each pattern, we can show the above statement by applying elementary deforamtion of type $\mathrm{II}^{+}$ once or twice. Suppose $\Sigma(A)$ is the surroundings of $A$. We may describe it as

$\Sigma(A)=\left\{B^{+} A F^{-}, C^{+} A G^{-}, D^{+} A H^{-}, B^{+} C^{+}, C^{+} D^{+}, D^{+} B^{+}, F^{-} G^{-}, G^{-} H^{-}, H^{-} F^{-}\right\}$.

Suppose $x$ and $y$ are an initial and a terminal 0-label of $A$. Since $x \neq y$, we can apply an elementary deformation $\Psi(A)$ of type $\mathrm{II}^{+}$to our 1-label $A$. Then we obtain

$$
\begin{aligned}
\Sigma_{*}= & P Q R ; B^{+} F^{-}, C^{+} G^{-}, D^{+} H^{-}, B^{+} R C^{+}, C^{+} P D^{+}, D^{+} Q B^{+}, \\
& \left.F^{-} R G^{-}, G^{-} P H^{-}, H^{-} Q F^{-}\right\} .
\end{aligned}
$$

Let $\Delta_{*}$ be the DS-diagram associated with $\Sigma_{*}$.

Pattern 1. Suppose $A$ is of $(3,3: 3,0)$. We may assume a good cycle $\Lambda$ of $\Delta$ is

$$
\Lambda: \cdots C^{+} A H^{-} \cdots D^{+} A F^{-} \cdots B^{+} A G^{-} \cdots \text {. }
$$

Then

$$
\Lambda_{*}: \cdots C^{+} P H^{-} \ldots D^{+} Q F^{-} \ldots B^{+} R G^{-} \ldots
$$

is obviously a good cycle of $\Delta_{*}$, where $b\left(\Delta_{*}\right)=k-1$ (Figure 9).

Pattern 2. Suppose $A$ is of $(4,3: 1,2)$. By $\Psi(A)$, a good cycle

$$
\Lambda: \cdots D^{+} A G^{-} \cdots D^{+} A H^{-} \ldots B^{-} A F^{-} \ldots D^{+} C^{+} \ldots
$$

of $\Delta$ is changes to

$$
\Lambda_{*}: \cdots D^{+} P^{-1} G^{-} \ldots D^{+} H^{-} \ldots B^{+} F^{-} \ldots D^{+} Q R C^{+} \ldots
$$

which is a good cycle of $\Delta_{*}$ with $b\left(\Delta_{*}\right)=k-1$ (Figure 10).

By the similar arguments, we can deal with patterns $(4,3: 2,1),(4,4: 0,3),(4,4$ : $1,2)$. For these patterns, we list here only the good cycles $\Lambda$ and $\Lambda_{*}$ of $\Delta$ and $\Delta_{*}$, where $b(\Delta)=k$ and $b\left(\Delta_{*}\right)=k-1$.

Pattern 3. $(4,3: 2,1)$.

$$
\begin{gathered}
\Lambda: \cdots D^{+} A G^{-} \cdots B^{+} A H^{-} \cdots B^{+} A F^{-} \cdots B^{+} C^{+} \ldots \\
\Lambda_{*}: \cdots D^{+} P G^{-} \cdots B^{+} Q H^{-} \cdots B^{+} F^{-} \cdots B^{+} Q^{-1} C^{+} \cdots
\end{gathered}
$$

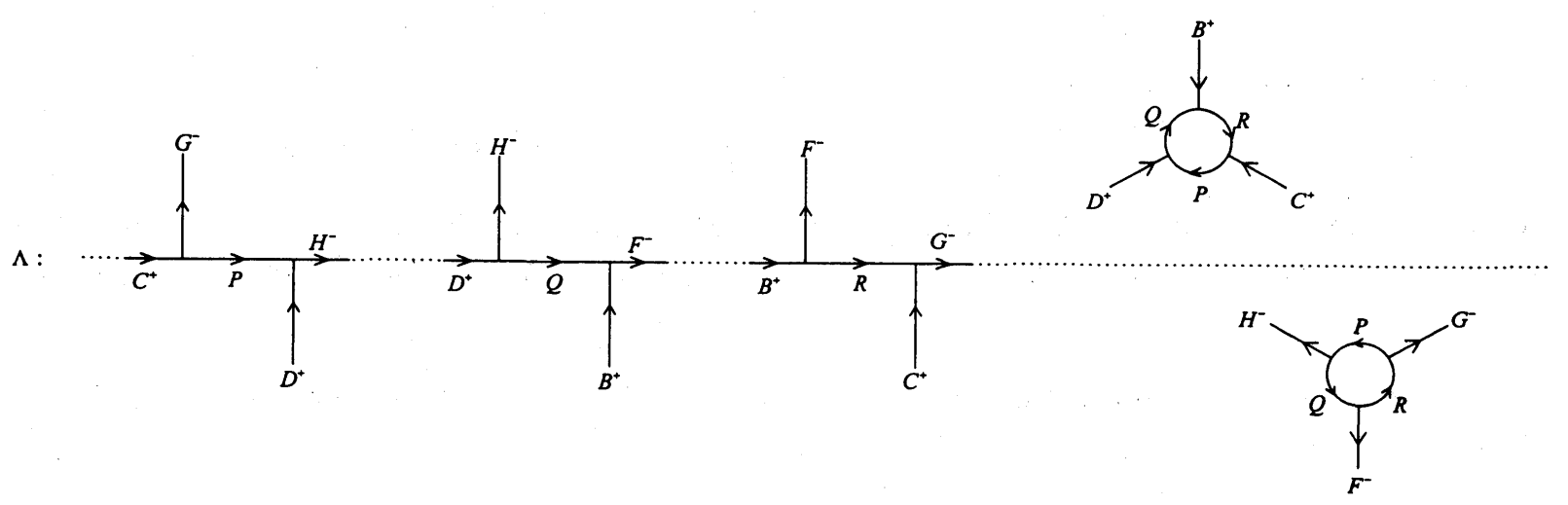

FIGURE 9. The case of pattern $(3,3: 3,0)$. 
$\Lambda$
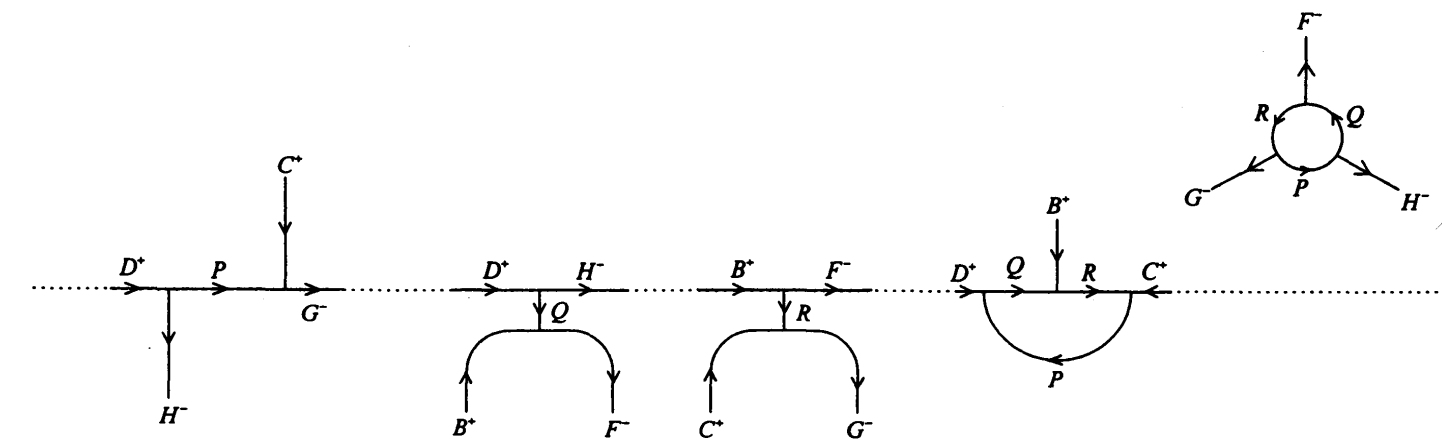

FIGURE 10. The case of pattern $(4,3: 1,2)$.

Pattern 4. $(4,4: 0,3)$.

$$
\begin{gathered}
\Lambda: \cdots D^{+} A H^{-} \ldots D^{+} A H^{-} \ldots B^{+} A F^{-} \ldots D^{+} C^{+} \ldots G^{-} H^{-} \ldots, \\
\Lambda_{*}: \cdots D^{+} H^{-} \ldots D^{+} H^{-} \cdots B^{+} F^{-} \ldots D^{+} P^{-1} C^{+} \ldots G^{-} R^{-1} Q^{-1} H^{-} \ldots .
\end{gathered}
$$

Pattern 5. $(4,4: 1,2)$.

$$
\begin{gathered}
\Lambda: \cdots C^{+} A H^{-} \ldots B^{+} A F^{-} \ldots B^{+} A F^{-} \ldots B^{+} D^{+} \ldots G^{-} F^{-} \ldots, \\
\Lambda_{*}: \cdots C^{+} P H^{-} \ldots B^{+} F^{-} \ldots B^{+} F^{-} \ldots B^{+} Q D^{+} \ldots G^{-} R F^{-} \ldots .
\end{gathered}
$$

Now we will deal with the remaining two patterns $(4,4: 2,1)$ and $(4,4: 3,0)$.

Pattern 6. Suppose $A$ is of $(4,4: 2,1) . \Lambda$ is written

$$
\Lambda: \cdots C^{+} A H^{-} \ldots B^{+} A H^{-} \cdots B^{+} A F^{-} \ldots B^{+} D^{+} \ldots G^{-} H^{-} \ldots .
$$

Applying $\Psi(A)$, we obtain a good cycle

$$
\Lambda^{\prime}: \cdots C^{+} P H^{-} \cdots B^{+} Q^{-1} H^{-} \cdots B^{+} F^{-} \cdots B^{+} R P D^{+} \ldots G^{-} P H^{-} \ldots
$$

of $\Delta^{\prime}$ (Figure 11-a); still $b\left(\Delta^{\prime}\right)=k$. But we can find a new bordered 1-label $P$ of pattern $(3,4: 2,1)$ in $\Lambda^{\prime}$. Hence we can apply $\Psi(P)$ to $\Delta^{\prime}$ again, and obtain $\Delta_{*}$ with $b\left(\Delta_{*}\right)=k-1$ (Figure 10-b) which has a good cycle $\Lambda_{*}$.

Pattern 7. Suppose $A$ is of $(4,4: 3,0) . \Lambda$ is written

$$
\Lambda: \cdots C^{+} A H^{-} \ldots D^{+} A F^{-} \ldots C^{+} A F^{-} \ldots C^{+} B^{+} \ldots G^{-} F^{-} \ldots .
$$

Applying $\Psi(A)$ to $\Lambda$, we obtain a good cycle

$$
\Lambda^{\prime}: \cdots C^{+} P H^{-} \ldots D^{+} Q F^{-} \ldots C^{+} R^{-1} F^{-} \ldots C^{+} R^{-1} B^{+} \ldots G^{-} R^{-1} F^{-} \ldots
$$

of another DS-diagram $\Delta^{\prime}$ such that $b\left(\Delta^{\prime}\right)=k . R$ is a new bordered 1-label of a pattern of $(4,4: 1,2)$. Again we apply $\Psi(R)$ to $\Delta^{\prime}$. Then we obtain $\Delta_{*}$ with a good cycle $\Lambda_{*}$ so that $b\left(\Delta_{*}\right)=k-1$.

By the induction on the number of the bordered 1-labels of seven patterns, we obtain the following corollary. 


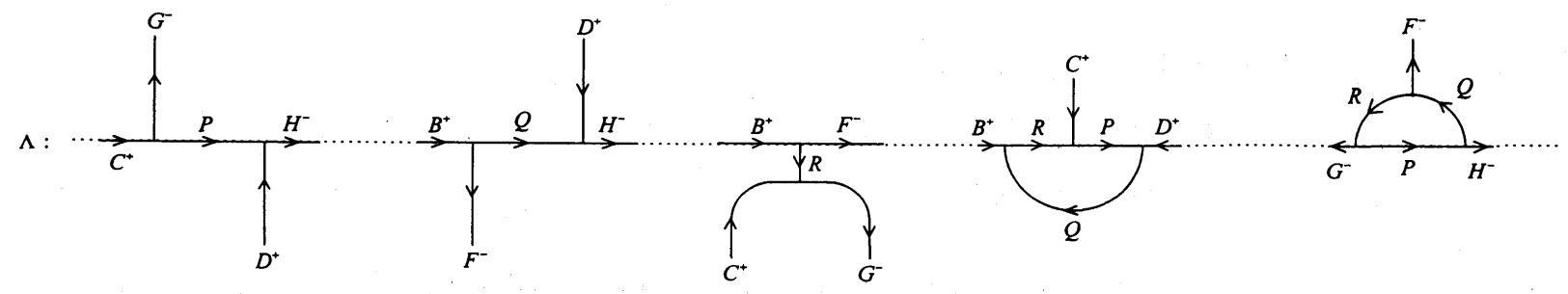

FIGURE 11-a. The case of pattern $(4,4: 2,1)$; first step.

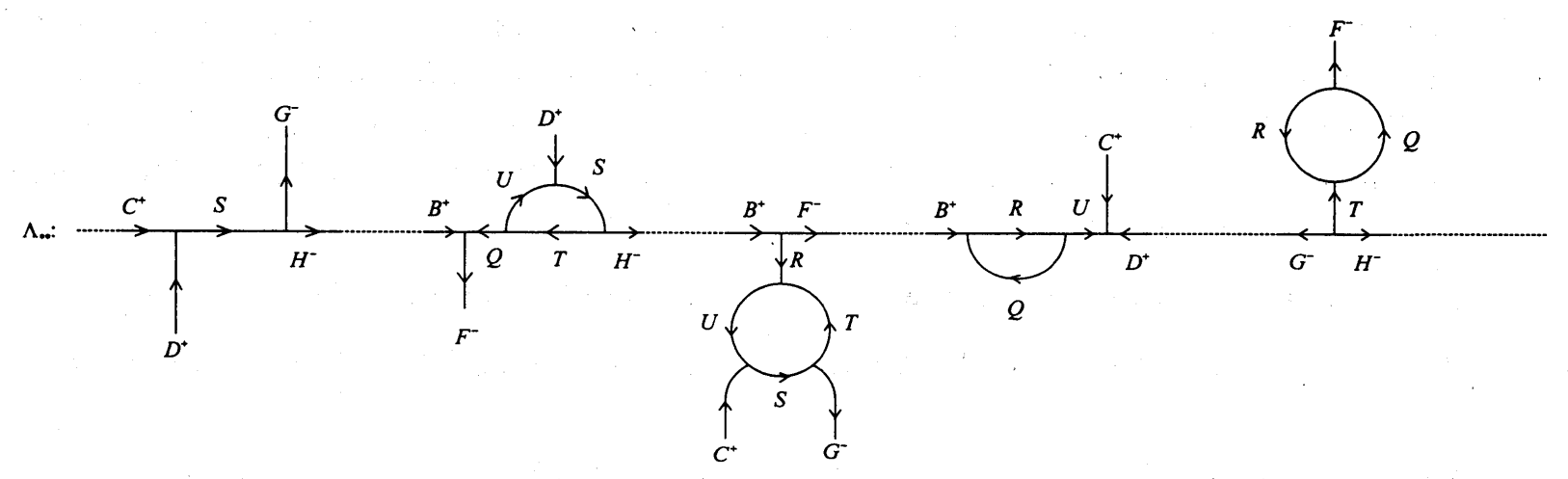

FIGURE 11-b. The case of pattern $(4,4: 2,1)$; second step.

COROLLARY 3.1. For any DS-diagram $\Delta$, there is a splittable DS-diagram $\Delta^{\prime}$ with a better cycle such that $\Delta^{\prime}$ is DS-isomorphic to $\Delta$.

\section{Remodeling into a DS-diagram with E-cycle.}

In the previous section, we saw that any DS-diagram can be remodeled into a splittable DS-diagram with a better cycle. In this section we will consider exclusively a splittable DSdiagram $\Delta$ with a better cycle $\Lambda$.

Remember that $b(\Delta)$ is the number of the bordered 1-labels of $\Delta$. The following theorem was found by Prof. Dr. Ippei Ishii.

THEOREM 4.1. The number $b(\Delta)$ is even, and the number of the bordered 1-labels of type $\left(U^{+}, U^{+}, U^{+}\right)$is equal to that of type $\left(U^{-}, U^{-}, U^{-}\right)$.

Proof. Let $\left(Z^{+}, Z^{-}\right)$be the bicoloring of $\Delta$. We will denote the number of positive (or negative) $i$-cell of $\Delta$ by $v^{+}(i)$ (or $v^{-}(i)$ ). By $v^{\circ}(i)$ we mean the number of the neutral $i$-cells. Then obviously $v^{+}(2)=v^{-}(2), v^{\circ}(2)=0$. And it holds that $v^{+}(1)=v^{-}(1)$ since 1-cells with a bordered 1-label are all neutral and three 1-cells with a distributed 1-label consists of one positive, one negative and one neutral 1-cell. Since both $Z^{+}$and $Z^{-}$are 2-disks, $\chi\left(Z^{+}\right)=\chi\left(Z^{-}\right)=1$, where $\chi\left(Z^{ \pm}\right)$is the Euler number of $Z^{ \pm}$. That is,

$$
\chi\left(Z^{ \pm}\right)=\left\{v^{\circ}(0)+v^{ \pm}(0)\right\}-\left\{v^{\circ}(1)+v^{ \pm}(1)\right\}+v^{ \pm}(2) \text {. }
$$


Hence $v^{+}(0)=v^{-}(0)$ follows from

$$
\begin{aligned}
0 & =\chi\left(Z^{+}\right)-\chi\left(Z^{-}\right) \\
& =\left\{v^{+}(0)-v^{-}(0)\right\}-\left\{v^{+}(1)-v^{-}(1)\right\}+\left\{v^{+}(2)-v^{-}(2)\right\} \\
& =v^{+}(0)-v^{-}(0)
\end{aligned}
$$

Suppose $m$ and $n$ are the number of the bordered 1-labels of type $\left(U^{+}, U^{+}, U^{+}\right)$and of type $\left(U^{-}, U^{-}, U^{-}\right)$respectively. Suppose $x$ is a 0-label. Let $v^{+}(x), v^{-}(x)$ and $v^{\circ}(x)$ be the number of positive, negative, and neutral 0-cells with the 0-label $x$, respectively. If each 1-label incident with $x$ is distributed, $v^{+}(x)=v^{-}(x)=1$ and $v^{\circ}(x)=2$. If there is a bordered 1-label, say $A$, incident with $x$, then there are no other bordered 1-labels incident with $x$. If $A$ is of type $\left(U^{+}, U^{+}, U^{+}\right)$, then $v^{\circ}(x)=3, v^{+}(x)=0$ and $v^{-}(x)=1$ holds. If $A$ is of type $\left(U^{-}, U^{-}, U^{-}\right)$, then $v^{\circ}(x)=3, v^{+}(x)=1$ and $v^{-}(x)=0$ holds. Therefore $v^{+}(0)-v^{-}(0)=n-m$. Hence $m=n$.

Showing the following theorem, we complete the proof of our main Theorem 1.1.

THEOREM 4.2. There is a DS-diagram $\Delta_{*}$ with E-cycle such that $\Delta_{*}$ is $D S$-isomorphic to $\Delta$.

PROOF. Suppose $b(\Delta)=2 k$. By the previous theorem, there is a pair of bordered 1-labels $X$ and $Y$ such that $X$ is of type $\left(U^{+}, U^{+}, U^{+}\right)$and $Y$ is of type $\left(U^{-}, U^{-}, U^{-}\right)$. We can assume

$$
\begin{aligned}
\Delta=\{ & \left\{C_{X}^{+} X G_{X}^{-}, D_{X}^{+} X H_{X}^{-}, B_{X}^{+} X F_{X}^{-},\right. \\
& C_{X}^{+} D_{X}^{+}, D_{X}^{+} B_{X}^{+}, B_{X}^{+} C_{X}^{+}, G_{X}^{-} H_{X}^{-}, H_{X}^{-} F_{X}^{-}, F_{X}^{-} G_{X}^{-}, \\
& C_{Y}^{+} Y G_{Y}^{-}, D_{Y}^{+} Y H_{Y}^{-}, B_{Y}^{+} Y F_{Y}^{-} \\
& \left.C_{Y}^{+} D_{Y}^{+}, D_{Y}^{+} B_{Y}^{+}, B_{Y}^{+} C_{Y}^{+}, G_{Y}^{-} H_{Y}^{-}, H_{Y}^{-} F_{Y}^{-}, F_{Y}^{-} G_{Y}^{-}, \cdots \cdots\right\},
\end{aligned}
$$

(see Figure 12-a), and

$$
\begin{aligned}
\Lambda= & \cdots D_{X}^{+} X H_{X}^{-} \cdots B_{X}^{+} X F_{X}^{-} \cdots C_{X}^{+} X G_{X}^{-} \cdots \\
& \cdots C_{Y}^{+} Y G_{Y}^{-} \cdots D_{Y}^{+} Y H_{Y}^{-} \cdots B_{Y}^{+} Y F_{Y}^{-} \cdots
\end{aligned}
$$

Applying an elementary deformation $\Psi(Y)$ of type $\mathrm{II}^{+}$on $\Delta$, we can obtain a DS-diagram $\Delta_{1}$ (see Figure 12-b) such that

$$
\begin{aligned}
\Delta_{1}=\{ & P Q R ; C_{X}^{+} X G_{X}^{-}, D_{X}^{+} X H_{X}^{-}, B_{X}^{+} X F_{X}^{-}, \\
& C_{X}^{+} D_{X}^{+}, D_{X}^{+} B_{X}^{+}, B_{X}^{+} C_{X}^{+}, G_{X}^{-} H_{X}^{-}, H_{X}^{-} F_{X}^{-}, F_{X}^{-} G_{X}^{-}, \\
& C_{Y}^{+} P D_{Y}^{+}, D_{Y}^{+} Q B_{Y}^{+}, B_{Y}^{+} R C_{Y}^{+}, G_{Y}^{-} P H_{Y}^{-}, H_{Y}^{-} Q F_{Y}^{-}, F_{Y}^{-} R G_{Y}^{-}, \\
& \left.C_{Y}^{+} G_{Y}^{-}, D_{Y}^{+} H_{Y}^{-}, B_{Y}^{+} F_{Y}^{-}, \cdots\right\} .
\end{aligned}
$$




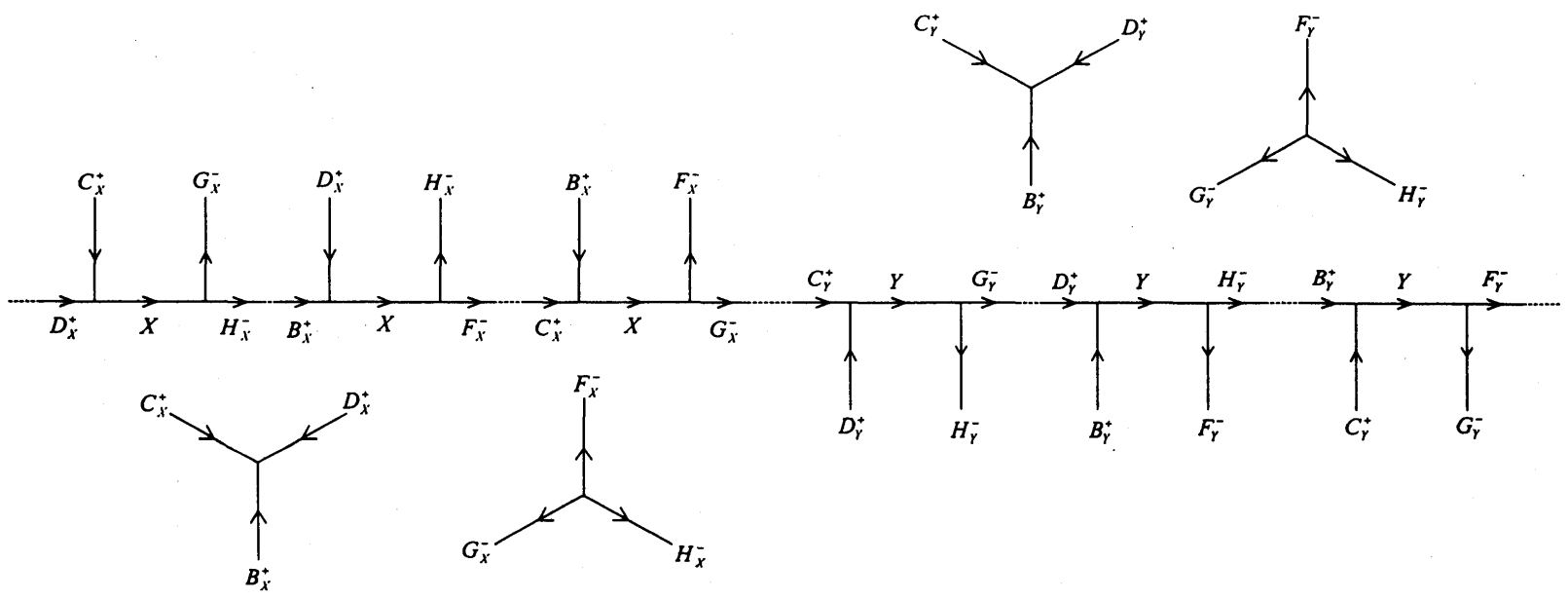

FIgURE 12-a. Pair of 1-labels $X$ and $Y ; X$ is of type $\left(U^{+}, U^{+}, U^{+}\right)$and $Y$ is of type $\left(U^{-}, U^{-}, U^{-}\right)$.

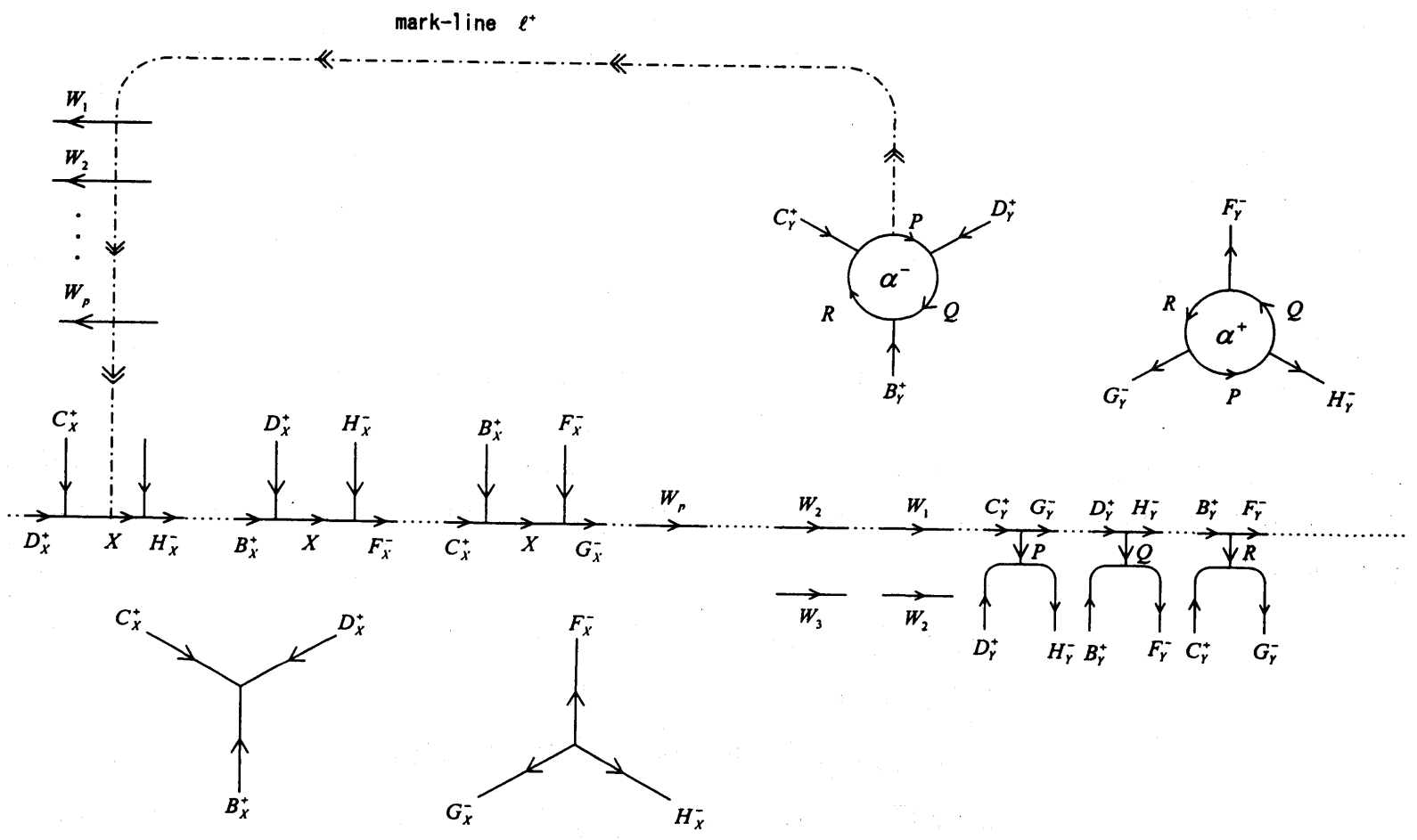

FIGURE 12-b. After applying an elementary deformation $\Psi(Y)$.

Note that

$$
\begin{aligned}
\Lambda_{1}= & \cdots D_{X}^{+} X H_{X}^{-} \cdots B_{X}^{+} X F_{X}^{-} \cdots C_{X}^{+} X G_{X}^{-} \cdots \\
& \cdots C_{Y}^{+} G_{Y}^{-} \cdots D_{Y}^{+} H_{Y}^{-} \cdots B_{Y}^{+} F_{Y}^{-} \cdots
\end{aligned}
$$

is no longer a splitting cycle of $\Delta_{1}$. But $Z^{+}$is still connected, a new 1-label $P \subset Z^{+}$is distributed and a 1-label $X \subset Z^{+}$is still bordered. We can find a path $\ell^{+}$from $P$ to $X$ as 


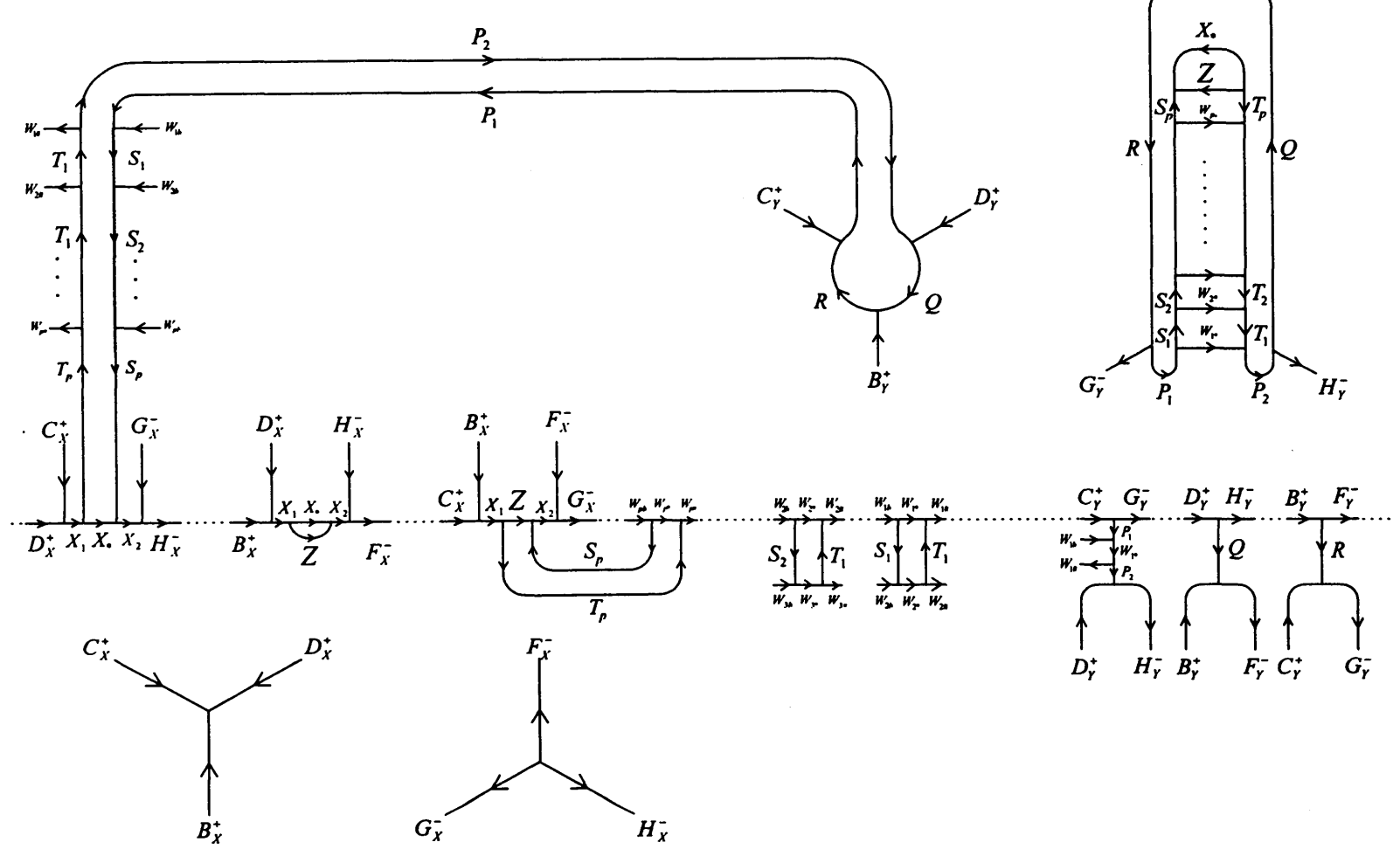

FIGURE 12-c. After applying a digging $d\left(\ell^{+}\right)$.

a mark line. $\ell^{+}$may cut across some 1-cells with 1-labels, say $W_{1}, W_{2}, \cdots, W_{p}$. Applying digging $d\left(\ell^{+}\right)$along $\ell^{+}$, we can obtain a splittable DS-diagram $\Delta_{2}$ (see Figure 12-c) with a good cycle

$$
\begin{aligned}
\Lambda_{2}= & \cdots D_{X}^{+} X_{1} T_{p} \cdots T_{2} T_{1} P_{2} Q R P_{1} S_{1} S_{2} \cdots S_{p} X_{2} H_{X}^{-} \cdots \\
& \cdots B_{X}^{+} X_{1} X_{*} X_{2} F_{X}^{-} \cdots C_{X}^{+} X_{1} Z X_{2} G_{X}^{-} \cdots \\
& \cdots W_{p \downarrow} W_{p *} W_{p \sharp} \cdots W_{2 b} W_{2 *} W_{2 \sharp} W_{1 \mathrm{~b}} W_{1 *} W_{1 \sharp} \cdots \\
& \cdots C_{Y}^{+} Y G_{Y}^{-} \cdots D_{Y}^{+} Y H_{Y}^{-} \cdots B_{Y}^{+} Y F_{Y}^{-} \cdots
\end{aligned}
$$

Note that both of $X_{1}$ and $X_{2}$ on $\Lambda_{2}$ are of pattern 2: (3, $\left.3: 3,0\right)$. Hence by applying $\Psi\left(X_{1}\right)$ and $\Psi\left(X_{2}\right)$, we can obtain a splittable DS-diagram $\Delta_{3}$ with a better cycle

$$
\begin{aligned}
\Lambda_{3}= & \cdots D_{X}^{+} J_{1}^{-1} T_{p} \cdots T_{2} T_{1} P_{2} Q R P_{1} S_{1} S_{2} \cdots S_{p} J_{2} H_{X}^{-} \cdots \\
& \cdots B_{X}^{+} K_{1}^{-1} X_{*} K_{2} F_{X}^{-} \cdots C_{X}^{+} L_{1}^{-1} Z L_{2} G_{X}^{-} \cdots \\
& \cdots W_{p \mathrm{~b}} W_{p *} W_{p \sharp} \cdots W_{2 \mathrm{~b}} W_{2 *} W_{2 \sharp} W_{1 \mathrm{~b}} W_{1 *} W_{1 \sharp} \cdots \\
& \cdots C_{Y}^{+} Y G_{Y}^{-} \cdots D_{Y}^{+} Y H_{Y}^{-} \cdots B_{Y}^{+} Y F_{Y}^{-} \cdots
\end{aligned}
$$


It is an easy observation that $b\left(\Delta_{3}\right)=2 k-2$. This argument shows that there is an algorithm to obtain a DS-diagram with E-cycle which is DS-isomorphic to the original $\Delta$.

\title{
References
}

[ 1 ] B. G. CASler, An embedding theorem for connected 3-manifolds with boundary, Proc. Amer. Math. Soc. 16 (1965), 559-566.

[ 2 ] H. IKEDA, Acyclic fake surfaces, Topology 10 (1971), 9-36.

[ 3 ] H. IKEDA, Identification maps on the 2-sphere, Kobe J. Math. 2 (1985), 163-167.

[ 4 ] H. IKEDA, DS-diagrams with E-cycle, Kobe J. Math. 3 (1986), 103-112.

[ 5 ] H. IKEDA and Y. INOUE, Invitation to DS-diagrams, Kobe J. Math. 2 (1985), 169-185.

[ 6 ] H. IKeda, M. Yamashita and K. Yokoyama, Symbolic description of homeomorphism on closed 3manifolds, Kobe J. Math. 13 (1996), 69-115.

[ 7 ] I. IsHII, Flows and spines, Tokyo J. Math. 9 (1986), 505-525.

[ 8 ] I. IsHII, Combinatorial construction of a non-singular flow on a 3-manifold, Kobe J. Math. 3 (1986), 201-208.

[ 9 ] A. T. Lundell and S. Weingram, The Topology of CW Complexes, Van Nostrand (1969).

\author{
Present Address: \\ HIROSHI IKEDA \\ DEPARTMENT OF MATHEMATICS, FACULTY OF SCIENCE, \\ KOBE UNIVERSITY, \\ KOBE 657-8501 JAPAN. \\ MASAKATSU YAMASHITA \\ COURSE OF GENERAL EDUCATION, FACULTY OF ENGINEERING, \\ TOYO UNIVERSITY, \\ KAWAGOE-SHI, SAITAMA, 350-8585 JAPAN. \\ KAZUO YOKOYAMA \\ Department of MAThematics, FACUlty of SCIENCE AND TEChNOlogy, \\ SOPHIA UNIVERSITY, \\ CHIYODA-KU, TOKYO, 102-8554 JAPAN.
}

